Department of Computer Science Technical Report

\title{
Bisimulation and Logical Preservation for Continuous-Time Markov Decision Processes
}

Martin Neuhäußer and Joost-Pieter Katoen

ISSN 0935-3232

RWTH Aachen
Aachener Informatik Berichte

Department of Computer Science
AIB-2007-10

August 2007 
The publications of the Department of Computer Science of $R W T H$ Aachen University are in general accessible through the World Wide Web.

http://aib.informatik.rwth-aachen.de/ 


\title{
Bisimulation and Logical Preservation for Continuous-Time Markov Decision Processes
}

\author{
Martin R. Neuhäußer ${ }^{1,2}$ and Joost-Pieter Katoen ${ }^{1,2}$ \\ 1 Software Modeling and Verification Group \\ RWTH Aachen University, Germany \\ 2 Formal Methods and Tools Group \\ University of Twente, The Netherlands \\ \{neuhaeusser, katoen\}@cs.rwth-aachen.de
}

\begin{abstract}
This paper introduces strong bisimulation for continuous-time Markov decision processes (CTMDPs), a stochastic model which allows for a nondeterministic choice between exponential distributions, and shows that bisimulation preserves the validity of CSL. To that end, we interpret the semantics of CSL-a stochastic variant of CTL for continuous-time Markov chains-on CTMDPs and show its measure-theoretic soundness. The main challenge faced in this paper is the proof of logical preservation that is substantially based on measure theory.
\end{abstract}

\section{Introduction}

Discrete-time probabilistic models, in particular Markov decision processes (MDP) [20], are used in various application areas such as randomized distributed algorithms and security protocols. A plethora of results in the field of concurrency theory and verification are known for MDPs. Efficient model-checking algorithms exist for probabilistic variants of CTL [9,11], linear-time [29] and long-run properties [15], process algebraic formalisms for MDPs have been developed and bisimulation is used to minimize MDPs prior to analysis [18].

In contrast, CTMDPs [25], a continuous-time variant of MDPs, where state residence times are exponentially distributed, have received scant attention. Unlike in MDPs, where nondeterminism occurs between discrete probability distributions, in CTMDPs the choice between various exponential distributions is nondeterministic. In case all exponential delays are uniquely determined, a continuous-time Markov chain (CTMC) results, a widely studied model in performance and dependability analysis.

This paper proposes strong bisimulation on CTMDPs - this notion is a conservative extension of bisimulation on CTMCs [13] — and investigates which kind of logical properties this preserves. In particular, we show that bisimulation preserves the validity of CSL $[3,5]$, a well-known logic for CTMCs. To that end, we provide a semantics of CSL on CTMDPs which is in fact obtained in a similar way as the semantics of PCTL on MDPs $[9,11]$. We show the semantic soundness of the logic using measure-theoretic arguments, and prove that bisimilar states preserve full CSL. Although this result is perhaps not surprising, its proof is non-trivial and strongly relies on measure-theoretic aspects. It shows that reasoning about CTMDPs, as witnessed also by $[30,7,10]$ is not straightforward. As for MDPs, CSL equivalence does not coincide with bisimulation as only maximal and minimal probabilities can be logically expressed.

Apart from the theoretical contribution, we believe that the results of this paper have wider applicability. CTMDPs are the semantic model of stochastic 
Petri nets [14] that exhibit confusion, stochastic activity networks [27] (where absence of nondeterminism is validated by a "well-specified" check), and is strongly related to interactive Markov chains which are used to provide compositional semantics to process algebras [19] and dynamic fault trees [12]. Besides, CTMDPs have practical applicability in areas such as stochastic scheduling $[17,1]$ and dynamic power management [26]. Our interest in CTMDPs is furthermore stimulated by recent results on abstraction - where the introduction of nondeterminism is the key principle of CTMCs [21] in the context of probabilistic model checking.

In our view, it is a challenge to study this continuous-time stochastic model in greater depth. This paper is a small, though important, step towards a better understanding of CTMDPs.

\section{Continuous-time Markov decision processes}

Continuous-time Markov decision processes extend continuous-time Markov chains by nondeterministic choices. Therefore each transition is labelled with an action referring to the nondeterministic choice and the rate of a negative exponential distribution which determines the transition's delay:

Definition 1 (Continuous-time Markov decision process). A tuple $\mathcal{C}=$ $(\mathcal{S}$, Act $, \mathbf{R}, A P, L)$ is a labelled continuous-time Markov decision process if $\mathcal{S}$ is a finite, nonempty set of states, Act a finite, nonempty set of actions and $\mathbf{R}: \mathcal{S} \times$ Act $\times \mathcal{S} \rightarrow \mathbb{R}_{\geq 0}$ a three-dimensional rate matrix. Further, AP is a finite set of atomic propositions and $L: \mathcal{S} \rightarrow 2^{A P}$ is a state labelling function.

The set of actions that are enabled in a state $s \in \mathcal{S}$ is denoted $\operatorname{Act}(s):=$ $\left\{\alpha \in\right.$ Act $\mid \exists s^{\prime} \in \mathcal{S}$. $\left.\mathbf{R}\left(s, \alpha, s^{\prime}\right)>0\right\}$. A CTMDP is well-formed if $\operatorname{Act}(s) \neq \emptyset$ for all $s \in \mathcal{S}$, that is, if every state has at least one outgoing transition. Note that this can easily be established for any CTMDP by adding self-loops.

Example 1. When entering state $s_{1}$ of the CTMDP in Fig. 1 (without state labels) one action from the set of enabled actions $\operatorname{Act}\left(s_{1}\right)=\{\alpha, \beta\}$ is chosen nondeterministically, say $\alpha$. Next, the rate of the $\alpha$-transition determines its exponentially distributed delay. Hence for a single $\alpha$-transition, the probability to go from $s_{1}$ to $s_{3}$ within time $t$ is

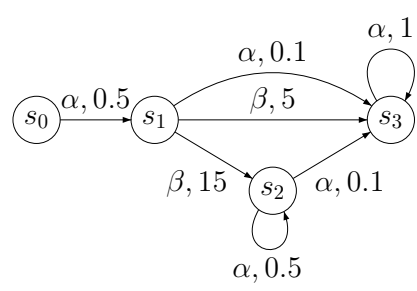

Fig. 1. Example of a CTMDP. $1-e^{-\mathbf{R}\left(s_{1}, \alpha, s_{3}\right) t}=1-e^{-0.1 t}$.

If multiple outgoing transitions exist for the chosen action, they compete according to their exponentially distributed delays: In Fig. 1 such a race condition occurs if action $\beta$ is chosen in state $s_{1}$. In this situation, two $\beta$-transitions (to $s_{2}$ and $\left.s_{3}\right)$ with rates $\mathbf{R}\left(s_{1}, \beta, s_{2}\right)=15$ and $\mathbf{R}\left(s_{1}, \beta, s_{3}\right)=5$ become available and state $s_{1}$ is left as soon as the first transition's delay expires. Hence the sojourn time in state $s_{1}$ is distributed according to the minimum of both exponential distributions, i.e. with rate $\mathbf{R}\left(s_{1}, \beta, s_{2}\right)+\mathbf{R}\left(s_{1}, \beta, s_{3}\right)=20$. In general, $E(s, \alpha):=\sum_{s^{\prime} \in \mathcal{S}} \mathbf{R}\left(s, \alpha, s^{\prime}\right)$ is the exit rate of state $s$ under action $\alpha$. Then $\mathbf{R}\left(s_{1}, \beta, s_{2}\right) / E\left(s_{1}, \beta\right)=0.75$ is the probability to move with $\beta$ from $s_{1}$ to $s_{2}$, i.e. the probability that the delay of the $\beta$-transition to $s_{2}$ expires first. Formally, 
the discrete branching probability is $\mathbf{P}\left(s, \alpha, s^{\prime}\right):=\frac{\mathbf{R}\left(s, \alpha, s^{\prime}\right)}{E(s, \alpha)}$ if $E(s, \alpha)>0$ and 0 otherwise. By $\mathbf{R}(s, \alpha, Q):=\sum_{s^{\prime} \in Q} \mathbf{R}\left(s, \alpha, s^{\prime}\right)$ we denote the total rate to states in $Q \subseteq \mathcal{S}$.

Definition 2 (Path). Let $\mathcal{C}=(\mathcal{S}, A c t, \mathbf{R}, A P, L)$ be a CTMDP. Paths ${ }^{n}(\mathcal{C}):=$ $\mathcal{S} \times\left(\text { Act } \times \mathbb{R}_{\geq 0} \times \mathcal{S}\right)^{n}$ is the set of paths of length $n$ in $\mathcal{C}$; the set of finite paths in $\mathcal{C}$ is defined by Paths ${ }^{\star}(\mathcal{C})=\bigcup_{n \in \mathbb{N}}$ Paths $^{n}$ and Paths ${ }^{\omega}(\mathcal{C}):=\left(\mathcal{S} \times \text { Act } \times \mathbb{R}_{\geq 0}\right)^{\omega}$ is the set of infinite paths in $\mathcal{C}$. Paths $(\mathcal{C}):=$ Paths $^{\star}(\mathcal{C}) \cup$ Paths ${ }^{\omega}(\mathcal{C})$ denotes the set of all paths in $\mathcal{C}$.

We write Paths instead of Paths $(\mathcal{C})$ whenever $\mathcal{C}$ is clear from the context. Paths are denoted $\pi=s_{0} \stackrel{\alpha_{0}, t_{0}}{\longrightarrow} s_{1} \stackrel{\alpha_{1}, t_{1}}{\longrightarrow} \cdots \stackrel{\alpha_{n-1}, t_{n-1}}{\longrightarrow} s_{n}$ where $|\pi|$ is the length of $\pi$. Given a finite path $\pi \in$ Paths $^{n}, \pi \downarrow$ is the last state of $\pi$. For $n<|\pi|, \pi[n]:=s_{n}$ is the $n$-th state of $\pi$ and $\delta(\pi, n):=t_{n}$ is the time spent in state $s_{n}$. Further, $\pi[i . . j]$ is the path-infix $s_{i} \stackrel{\alpha_{i}, t_{i}}{\longrightarrow} s_{i+1} \stackrel{\alpha_{i+1}, t_{i+1}}{\longrightarrow} \cdots \stackrel{\alpha_{j-1}, t_{j-1}}{\longrightarrow} s_{j}$ of $\pi$ for $i<j \leq|\pi|$. Finally, $\pi @ t$ is the state occupied in $\pi$ at time point $t \in \mathbb{R}_{\geq 0}$, i.e. $\pi @ t:=\pi[n]$ where $n$ is the smallest index such that $\sum_{i=0}^{n} t_{i}>t$.

Note that Def. 2 does not impose any semantic restrictions on paths. The set Paths in general contains paths which do not comply with the rate matrix of the underlying CTMDP. However, the following definition of the probability measure (Def. 4) justifies this as it assigns probability zero to such sets of paths.

\subsection{The probability space}

In probability theory (see [2]), a field of sets $\mathfrak{F} \subseteq 2^{\Omega}$ is a family of subsets of a set $\Omega$ which contains the empty set and is closed under complement and finite union. A field $\mathfrak{F}$ is a $\sigma$-field $d^{3}$ if it is also closed under countable union, i.e. if for all countable families $\left\{A_{i}\right\}_{i \in I}$ of sets $A_{i} \in \mathfrak{F}$ it holds $\bigcup_{i \in I} A_{i} \in \mathfrak{F}$. Any subset $A$ of $\Omega$ which is in $\mathfrak{F}$ is called measurable.

To measure the probability of sets of paths, we first define a $\sigma$-field of sets of combined transitions which we later use to define $\sigma$-fields of sets of finite and infinite paths. Here, a combined transition is a tuple $(\alpha, t, s)$ which links the decision for action $\alpha$ (which is given by a scheduler, see Def. 3 ) with the exponentially distributed time-point $t$ to move to a successor state $s$ of the underlying CTMDP. Formally, for a CTMDP $\mathcal{C}=(\mathcal{S}$, Act, $\mathbf{R}, A P, L)$, the set of combined transitions is $\Omega=A c t \times \mathbb{R}_{\geq 0} \times \mathcal{S}$. To define a probability space on $\Omega$, note that $\mathcal{S}$ and Act are finite; hence, the corresponding $\sigma$-fields are defined as $\mathfrak{F}_{\text {Act }}:=2^{\text {Act }}$ and $\mathfrak{F}_{\mathcal{S}}:=2^{\mathcal{S}}$. Any combined transition occurs at some time point $t \in \mathbb{R}_{\geq 0}$, so that we can use the Borel $\sigma$-field $\mathfrak{B}\left(\mathbb{R}_{\geq 0}\right)$ to measure the corresponding subsets of $\mathbb{R}_{\geq 0}$. In the following, we denote the sets of probability distributions on $\mathfrak{F}_{A c t}$ and $\mathfrak{F}_{\mathcal{S}}$ by $\operatorname{Distr}(A c t)$ and $\operatorname{Distr}(\mathcal{S})$, respectively. Note that any path $\pi=s_{0} \stackrel{\alpha_{0}, t_{0}}{\longrightarrow} s_{1} \stackrel{\alpha_{1}, t_{1}}{\longrightarrow} \cdots \stackrel{\alpha_{n-1}, t_{n-1}}{\longrightarrow} s_{n}$ of length $n$ can be extended by a combined transition $m=\left(\alpha_{n}, t_{n}, s_{n+1}\right)$ to a path of length $n+1$, denoted $\pi \circ m$.

Generally, a Cartesian product is a measurable rectangle if its constituent sets are elements of their respective $\sigma$-fields. For example, in our case the set $A \times T \times S$ is a measurable rectangle if $A \in \mathfrak{F}_{A c t}, T \in \mathfrak{B}\left(\mathbb{R}_{\geq 0}\right)$ and $S \in \mathfrak{F}_{\mathcal{S}}$.

\footnotetext{
${ }^{3}$ In the literature [22], $\sigma$-fields are also called $\sigma$-algebras.
} 
We use $\mathfrak{F}_{\text {Act }} \times \mathfrak{B}\left(\mathbb{R}_{\geq 0}\right) \times \mathfrak{F}_{\mathcal{S}}$ to denote the set of all measurable rectangles ${ }^{4}$. It generates the desired $\sigma$-field $\mathfrak{F}$ of sets of combined transitions, i.e. $\mathfrak{F}:=\sigma\left(\mathfrak{F}_{\text {Act }} \times\right.$ $\left.\mathfrak{B}\left(\mathbb{R}_{\geq 0}\right) \times \mathfrak{F}_{\mathcal{S}}\right)$.

Now $\mathfrak{F}$ may be used to infer the $\sigma$-fields $\mathfrak{F}_{\text {Paths }^{n}}$ of sets of paths of length $n$ : $\mathfrak{F}_{\text {Paths }^{n}}$ is generated by the set of measurable (path) rectangles, i.e. $\mathfrak{F}_{\text {Paths }^{n}}:=$ $\sigma\left(\left\{S_{0} \times M_{0} \times \cdots \times M_{n} \mid S_{0} \in \mathfrak{F}_{\mathcal{S}}, M_{i} \in \mathfrak{F}, 0 \leq i \leq n\right\}\right)$. Intuitively, $\mathfrak{F}_{\text {Paths }^{n}}$ consists of all possible (even countable infinite) unions and intersections of measurable path rectangles.

Example 2. For the CTMDP in Fig. 1, the event "from $s_{1}$ we directly reach state $s_{3}$ within 0.5 time units" and the event "if action $\alpha$ is chosen in state $s_{1}$, we remain in $s_{1}$ for less than 0.2 or more than 1 time units" are described by the Cartesion products $\Pi_{1}=\left\{s_{1}\right\} \times$ Act $\times[0,0.5] \times\left\{s_{3}\right\}$ and $\Pi_{2}=\left\{s_{1}\right\} \times\{\alpha\} \times$ $([0,0.2) \cup(1, \infty)) \times \mathcal{S} . \Pi_{1}$ and $\Pi_{2}$ are measurable rectangles whereas their union $\Pi_{1} \cup \Pi_{2}$ is an element of the $\sigma$-field $\mathfrak{F}_{\text {Paths }}{ }^{2}$.

The $\sigma$-field of sets of infinite paths is obtained using the cylinder-set construction [2]: A set $C^{n}$ of paths of length $n$ is called a cylinder base; it induces the infinite cylinder $C_{n}=\left\{\pi \in\right.$ Paths $\left.{ }^{\omega} \mid \pi[0 . . n] \in C^{n}\right\}$. A cylinder $C_{n}$ is measurable if $C^{n} \in \mathfrak{F}_{\text {Paths }^{n}} ; C_{n}$ is called an infinite rectangle if $C^{n}=S_{0} \times A_{0} \times T_{0} \times \cdots \times$ $A_{n-1} \times T_{n-1} \times S_{n}$ and $S_{i} \subseteq \mathcal{S}, A_{i} \subseteq$ Act and $T_{i} \subseteq \mathbb{R}_{\geq 0}$. It is a measurable infinite rectangle, if $S_{i} \in \mathfrak{F}_{\mathcal{S}}, A_{i} \in \mathfrak{F}_{A c t}$ and $T_{i} \in \mathfrak{B}\left(\mathbb{R}_{\geq 0}\right)$. We obtain the desired $\sigma$-field of sets of infinite paths as the minimal $\sigma$-field generated by the set of measurable cylinders; formally: $\mathfrak{F}_{\text {Paths }^{\omega}}:=\sigma\left(\bigcup_{n=0}^{\infty}\left\{C_{n} \mid C^{n} \in \mathfrak{F}_{\text {Paths }^{n}}\right\}\right)$.

Finally, the $\sigma$-field $\mathfrak{F}_{\text {Paths }}{ }^{\star}$ over finite and infinite paths is the smallest $\sigma$-field generated by the disjoint union $\bigcup_{n=0}^{\infty} \mathfrak{F}_{\text {Paths }^{n}} \cup \mathfrak{F}_{\text {Paths }^{\omega}}$.

\subsection{The probability measure}

To define a semantics for CTMDPs we use schedulers ${ }^{5}$ to resolve the nondeterministic choices. Thereby we obtain probability measures on the probability spaces defined above. A scheduler quantifies the probability of the next action based on the history of the system: If state $s$ is reached via finite path $\pi$, the scheduler yields a probability distribution over $\operatorname{Act}(\pi \downarrow)$. The type of schedulers we use is the class of measurable timed history-dependent randomized schedulers [30]:

Definition 3 (Measurable scheduler). Let $\mathcal{C}$ be a CTMDP with action set Act. A mapping $\mathcal{D}:$ Paths ${ }^{\star} \times \mathfrak{F}_{\text {Act }} \rightarrow[0,1]$ is a measurable scheduler if $\mathcal{D}(\pi, \cdot) \in$ $\operatorname{Distr}(\operatorname{Act}(\pi \downarrow))$ for all $\pi \in$ Paths $^{\star}$ and the functions $\mathcal{D}(\cdot, A):$ Paths $^{\star} \rightarrow[0,1]$ are measurable for all $A \in \mathfrak{F}_{\text {Act }}$. THR denotes the set of measurable schedulers.

In Def. 3, the measurability condition states that for any $B \in \mathfrak{B}([0,1])$ and $A \in \mathfrak{F}_{\text {Act }}$ the set $\left\{\pi \in\right.$ Paths $\left.^{\star} \mid \mathcal{D}(\pi, A) \in B\right\} \in \mathfrak{F}_{\text {Paths }^{\star}}$, see [30]. In the following, note that $\mathcal{D}(\pi, \cdot)$ is a probability measure with support $\subseteq \operatorname{Act}(\pi \downarrow)$; further $\mathbf{P}(s, \alpha, \cdot) \in \operatorname{Distr}(\mathcal{S})$ if $\alpha \in \operatorname{Act}(s)$. Let $\eta_{E(\pi \downarrow, \alpha)}(t):=E(\pi \downarrow, \alpha) \cdot e^{-E(\pi \downarrow, \alpha) t}$ denote the probability density function of the negative exponential distribution with parameter $E(\pi \downarrow, \alpha)$.

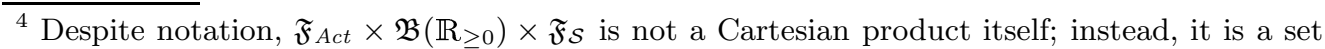
of Cartesian products.

${ }^{5}$ Schedulers are also called policies, strategies or adversaries in the literature.
} 
To derive a probability measure on $\mathfrak{F}_{\text {Paths }}{ }^{\omega}$, we first define a probability measure on combined transitions, i.e. on the measurable space $(\Omega, \mathfrak{F})$ : For history $\pi \in$ Paths $^{\star}$, let $\mu_{\mathcal{D}}(\pi, \cdot): \mathfrak{F} \rightarrow[0,1]$ such that

$$
\mu_{\mathcal{D}}(\pi, M):=\int_{A c t} \mathcal{D}(\pi, d \alpha) \int_{\mathbb{R}_{\geq 0}} \eta_{E(\pi \downarrow, \alpha)}(d t) \int_{\mathcal{S}} \mathbf{I}_{M}(\alpha, t, s) \mathbf{P}(\pi \downarrow, \alpha, d s) .
$$

Then $\mu_{\mathcal{D}}(\pi, \cdot)$ defines a probability measure on $\mathfrak{F}$ where the indicator function $\mathbf{I}_{M}(\alpha, t, s):=1$ if the combined transition $(\alpha, t, s) \in M$ and 0 otherwise [30]. Intuitively, for a given finite path $\pi$ and a set $M$ of combined transitions, $\mu_{\mathcal{D}}(\pi, M)$ is the probability to continue from $\pi \downarrow$ by one of the combined transitions in $M$. For a measurable rectangle $A \times T \times S^{\prime} \in \mathfrak{F}$ and time interval $T$, we obtain

$$
\mu_{\mathcal{D}}\left(\pi, A \times T \times S^{\prime}\right)=\sum_{\alpha \in A} \mathcal{D}(\pi,\{\alpha\}) \cdot \mathbf{P}\left(\pi \downarrow, \alpha, S^{\prime}\right) \cdot \int_{T} E(\pi \downarrow, \alpha) \cdot e^{-E(\pi \downarrow, \alpha) t} d t
$$

which is the probability to leave $\pi \downarrow$ via some action in $A$ within time interval $T$ to a state in $S^{\prime}$.

Lemma 1. For any $\pi \in$ Paths $^{\star}$, the function $\mu_{\mathcal{D}}(\pi, \cdot): \mathfrak{F} \rightarrow[0,1]$ is a probability measure on $(\Omega, \mathfrak{F})$.

Proof. This follows from [2, Theorem 2.6.7], for $\mathcal{D}(\pi, \cdot)$ is a probability measure and all $\eta_{E(\pi \downarrow, \alpha)}$ as well as $\mathbf{P}(\pi \downarrow, \alpha, \cdot)$ are probability measures for $\alpha \in A c t(\pi \downarrow)$.

To extend this to a probability measure on $\mathfrak{F}_{P_{a t h s}{ }^{n}}$, we assume an initial distribution $\nu \in \operatorname{Distr}(\mathcal{S})$ for the probability to start in a certain state $s$ and inductively append sets of combined transitions. To ease notation, we write $\nu(s)$ instead of $\nu(\{s\})$ where appropriate.

As the probability measures in Def. 4 (see below) depend on the Lebesgue integral of a function involving the measure $\mu_{\mathcal{D}}$, we have to show that $\mu_{\mathcal{D}}$ : Paths $^{\star} \times \mathfrak{F} \rightarrow[0,1]$ is measurable in its first argument, i.e. that for all $M \in \mathfrak{F}$ and $B \in \mathfrak{B}([0,1])$ it is the case that $\mu_{\mathcal{D}}(\cdot, M)^{-1}(B) \in \mathfrak{F}_{\text {Paths }^{\star}}$. The following theorem stems from Wolovick and Johr in [30] and is restated here only for the sake of completeness:

Theorem 1 (Combined transition measurability [30, Theorem 1]). Let $\mathcal{C}$ be a CTMDP with set Act of actions and $\mathcal{D}$ a scheduler. For all $A \in \mathfrak{F}_{\text {Act }}$, it holds: $\mathcal{D}(\cdot, A):$ Paths $^{\star} \rightarrow[0,1]$ is measurable iff $\forall M \in \mathfrak{F}, \mu_{\mathcal{D}}(\cdot, M):$ Paths ${ }^{\star} \rightarrow$ $[0,1]$ is measurable.

Hence $\mu_{\mathcal{D}}:$ Paths $^{\star} \times \mathfrak{F} \rightarrow[0,1]$ is measurable in its first argument whenever $\mathcal{D}$ is a measurable scheduler as defined in Def. 3. Note also, that the restriction $\mu_{\mathcal{D}}:$ Paths $^{n} \times \mathfrak{F} \rightarrow[0,1]$ is measurable w.r.t. $\mathfrak{F}_{\text {Path }^{n}}$.

With this precondition satisfied, we can define the probability measure on sets of finite paths as follows: 
Definition 4 (Probability measure [30]). For initial distribution $\nu \in \operatorname{Distr}(\mathcal{S})$ the probability measure on $\mathfrak{F}_{\text {Paths }^{n}}$ is defined inductively:

$$
\begin{aligned}
& \operatorname{Pr}_{\nu, \mathcal{D}}^{0}: \mathfrak{F}_{\text {Paths }^{0}} \rightarrow[0,1]: \Pi \mapsto \sum_{s \in \Pi} \nu(s) \quad \text { and for } n>0 \\
& \operatorname{Pr}_{\nu, \mathcal{D}}^{n}: \mathfrak{F}_{\text {Paths }^{n}} \rightarrow[0,1]: \Pi \mapsto \int_{\text {Paths }^{n-1}} \operatorname{Pr}_{\nu, \mathcal{D}}^{n-1}(d \pi) \int_{\Omega} \mathbf{I}_{\Pi}(\pi \circ m) \mu_{\mathcal{D}}(\pi, d m) .
\end{aligned}
$$

One further remark might be in order: For $n>0$, the Lebesgue integral in Def. 4 is well defined as the functions

$$
f_{\Pi}: \text { Paths }^{n-1} \rightarrow[0,1]: \pi \mapsto \int_{\Omega} \mathbf{I}_{\Pi}(\pi \circ m) \mu_{\mathcal{D}}(\pi, d m)
$$

are measurable for all $\Pi \in \mathfrak{F}_{\text {Paths }^{n}}$. First, $\{m \in \Omega \mid \pi \circ m \in \Pi\} \in \mathfrak{F}$ for all $\pi \in$ Paths $^{n-1}$ : If $\Pi=S_{0} \times M_{0} \times \cdots \times M_{n-1}$ is a measurable rectangle such that $M_{i} \in \mathfrak{F}$ for $0 \leq i<n$, we obtain

$$
\{m \in \Omega \mid \pi \circ m \in \Pi\}= \begin{cases}M_{n-1} & \text { if } \pi \in S_{0} \times M_{0} \times \cdots \times M_{n-2} \\ \emptyset & \text { otherwise. }\end{cases}
$$

Hence, for measurable rectangle $\Pi$, the set $\{m \in \Omega \mid \pi \circ m \in \Pi\}$ is measurable. Now, let $\Pi=\Pi_{1} \cup \Pi_{2}$ and $M_{i}=\left\{m \in \Omega \mid \pi \circ m \in \Pi_{i}\right\}$ for $i=1,2$. By induction hypothesis, $M_{i} \in \mathfrak{F}$; further, $\{m \in \Omega \mid \pi \circ m \in \Pi\}=M_{1} \cup M_{2}$. As $\mathfrak{F}$ is closed under countable union, $M_{1} \cup M_{2} \in \mathfrak{F}$. For the complement $\Pi^{c}$, define $M=\{m \in \Omega \mid \pi \circ m \in \Pi\}$. By induction hypothesis, $M \in \mathfrak{F}$. Further observe that $\left\{m \in \Omega \mid \pi \circ m \in \Pi^{c}\right\}=\{m \in \Omega \mid \pi \circ m \notin \Pi\}=\{m \in \Omega \mid \pi \circ m \in \Pi\}^{c}=$ $M^{c}$. Then $M^{c} \in \mathfrak{F}$ follows since $M \in \mathfrak{F}$ and $\mathfrak{F}$ is closed under complement. Now the functions $f_{\Pi}$ can be restated as follows:

$$
f_{\Pi}: \text { Paths }^{n-1} \rightarrow[0,1]: \pi \mapsto \mu_{\mathcal{D}}(\pi,\{m \in \Omega \mid \pi \circ m \in \Pi\})
$$

which is measurable w.r.t. $\mathfrak{F}_{\text {Paths }}{ }^{n-1}$ by Theorem 1 , where $\mu_{\mathcal{D}}$ is restricted to Paths ${ }^{n-1}$.

By Def. 4 we obtain measures on all $\sigma$-fields $\mathfrak{F}_{\text {Paths }^{n}}$. This extends to a measure on $\left(\right.$ Paths $\left.{ }^{\omega}, \mathfrak{F}_{\text {Paths }}{ }^{\omega}\right)$ as follows: First, note that any measurable cylinder can be represented by a base of finite length, i.e. $C_{n}=\left\{\pi \in\right.$ Paths $\left.^{\omega} \mid \pi[0 . . n] \in C^{n}\right\}$. Now the measures $\operatorname{Pr}_{\nu, \mathcal{D}}^{n}$ on $\mathfrak{F}_{\text {Paths }^{n}}$ extend to a unique probability measure $\operatorname{Pr}_{\nu, \mathcal{D}}^{\omega}$ on $\mathfrak{F}_{\text {Paths }} \omega$ by defining $\operatorname{Pr}_{\nu, \mathcal{D}}^{\omega}\left(C_{n}\right)=\operatorname{Pr}_{\nu, \mathcal{D}}^{n}\left(C^{n}\right)$. Although any measurable rectangle with base $C^{m}$ can equally be represented by a higher-dimensional base (more precisely, if $m<n$ and $C^{n}=C^{m} \times \Omega^{n-m}$ then $C_{n}=C_{m}$ ), the IonescuTulcea extension theorem [2] is applicable due to the inductive definition of the measures $\operatorname{Pr}_{\nu, \mathcal{D}}^{n}$ and assures the extension to be well defined and unique.

Lemma 2. $\operatorname{Pr}_{\nu, \mathcal{D}}^{n}$ is a probability measure on $\left(\right.$ Paths $\left.^{n}, \mathfrak{F}_{\text {Paths }^{n}}\right)$ for all $n \in \mathbb{N}$.

Proof. By induction on $n . \nu$ is a probability measure on $\left(\mathcal{S}, \mathfrak{F}_{\mathcal{S}}\right)$ and so is $\operatorname{Pr}_{\nu, \mathcal{D}}^{0}$. For $n>0$,

$$
\operatorname{Pr}_{\nu, \mathcal{D}}^{n}(\Pi)=\int_{\operatorname{Paths}^{n-1}} \operatorname{Pr}_{\nu, \mathcal{D}}^{n-1}(d \pi) \int_{\Omega} \mathbf{I}_{\Pi}(\pi \circ m) \mu_{\mathcal{D}}(\pi, d m) .
$$

By the induction hypothesis, $\operatorname{Pr}_{\nu, \mathcal{D}}^{n-1}$ is a probability measure; the same holds for $\mu_{\mathcal{D}}(\pi, \cdot)$ by Lemma 1 . The induction step then follows by $[2,2.6 .2]$. 
Definition 4 inductively appends transition triples to the path prefixes of length $n$ to obtain a measure on sets of paths of length $n+1$. In the proof of Theorem 5, we use an equivalent characterization that constructs paths reversely, i.e. paths of length $n+1$ are obtained from paths of length $n$ by concatenating an initial triple from the set $\mathcal{S} \times A c t \times \mathbb{R}_{\geq 0}$ to the suffix of length $n$ :

Definition 5 (Initial triples). Let $\mathcal{C}=(\mathcal{S}, A c t, \mathbf{R}, A P, L)$ be a $C T M D P, \nu \in$

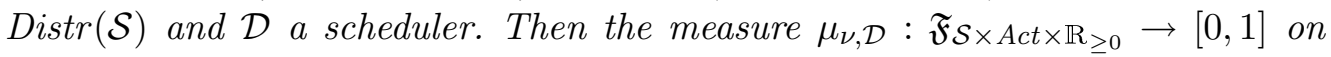
sets I of initial triples $(s, \alpha, t)$ is defined as

$$
\mu_{\nu, \mathcal{D}}(I)=\int_{\mathcal{S}} \nu(d s) \int_{A c t} \mathcal{D}(s, d \alpha) \int_{\mathbb{R}_{\geq 0}} \mathbf{I}_{I}(s, \alpha, t) \eta_{E(s, \alpha)}(d t) .
$$

This allows to decompose a path $\pi=s_{0} \stackrel{\alpha_{0}, t_{0}}{\longrightarrow} \cdots \stackrel{\alpha_{n-1}, t_{n-1}}{\longrightarrow} s_{n}$ into an initial triple $i=\left(s_{0}, \alpha_{0}, t_{0}\right)$ and the path suffix $\pi[1 . . n]$. For this to be measure preserving, a new $\nu_{i} \in \operatorname{Distr}(\mathcal{S})$ is defined based on the original initial distribution $\nu$ of $\operatorname{Pr}_{\nu, \mathcal{D}}^{n}$ on $\mathfrak{F}_{\text {Paths }^{n}}$ which reflects the fact that state $s_{0}$ has already been left with action $\alpha_{0}$ at time $t_{0}$. Hence $\nu_{i}$ is the initial distribution for the suffix-measure on $\mathfrak{F}_{\text {Paths }^{n-1}}$. Similarly, a scheduler $\mathcal{D}_{i}$ is defined which reproduces the decisions of the original scheduler $\mathcal{D}$ given that the first $i$-step is already taken. Hence $\operatorname{Pr}_{\nu_{i}, \mathcal{D}_{i}}^{n-1}$ is the adjusted probability measure on $\mathfrak{F}_{\text {Paths }}{ }^{n-1}$ given $\nu_{i}$ and $\mathcal{D}_{i}$.

Lemma 3. For $n \geq 1$ let $I \times \Pi \in \mathfrak{F}_{\text {Paths }^{n}}$ be a measurable rectangle, where $I \in \mathfrak{F}_{\mathcal{S}} \times \mathfrak{F}_{\text {Act }} \times \mathfrak{B}\left(\mathbb{R}_{\geq 0}\right)$. For $i=(s, \alpha, t) \in I$, let $\nu_{i}:=\mathbf{P}(s, \alpha, \cdot)$ and $\mathcal{D}_{i}(\pi):=$ $\mathcal{D}(i \circ \pi)$. Then $\operatorname{Pr}_{\nu, \mathcal{D}}^{n}(I \times \Pi)=\int_{I} \operatorname{Pr}_{\nu_{i}, \mathcal{D}_{i}}^{n-1}(\Pi) \mu_{\nu, \mathcal{D}}(d i)$.

Proof. By induction on $n$ :

For the induction start $(n=1)$, let $\Pi \in \mathfrak{F}_{\text {Paths }}$, i.e. $\Pi \subseteq \mathcal{S}$. Then:

$$
\begin{aligned}
& \operatorname{Pr}_{\nu, \mathcal{D}}^{1}(I \times \Pi)=\int_{\operatorname{Paths}^{\mathcal{O}}} \operatorname{Pr}^{0} \mathcal{D}(d \pi) \int_{\Omega} \mathbf{I}_{I \times \Pi}(\pi \circ m) \mu_{\mathcal{D}}(\pi, d m) \quad\left(* \text { Definition } 4{ }^{*}\right) \\
& =\int_{\mathcal{S}} \nu\left(d s_{0}\right) \int_{\Omega} \mathbf{I}_{I \times \Pi}\left(s_{0} \circ m\right) \mu_{\mathcal{D}}\left(s_{0}, d m\right) \quad \quad \quad\left(* \text { Paths }^{0}=\mathcal{S} *\right) \\
& =\int_{\mathcal{S}} \nu\left(d s_{0}\right) \int_{A c t} \mathcal{D}\left(s_{0}, d \alpha_{0}\right) \int_{\mathbb{R}_{\geq 0}} \eta_{E\left(s_{0}, \alpha_{0}\right)}\left(d t_{0}\right) \int_{\mathcal{S}} \mathbf{I}_{I \times \Pi}\left(s_{0} \stackrel{\alpha_{0}, t_{0}}{\longrightarrow} s_{1}\right) \mathbf{P}\left(s_{0}, \alpha_{0}, d s_{1}\right) \\
& \left.=\int_{I} \mu_{\nu, \mathcal{D}}\left(d s_{0}, d \alpha_{0}, d t_{0}\right) \int_{\mathcal{S}} \mathbf{I}_{\Pi}\left(s_{1}\right) \mathbf{P}\left(s_{0}, \alpha_{0}, d s_{1}\right) \quad \quad \text { (* definition of } \mu_{\nu, \mathcal{D}}{ }^{*}\right) \\
& =\int_{I} \mu_{\nu, \mathcal{D}}(d i) \int_{\mathcal{S}} \mathbf{I}_{\Pi}\left(s_{1}\right) \nu_{i}\left(d s_{1}\right) \quad\left(* i=\left(s_{0}, \alpha_{0}, t_{0}\right) *\right) \\
& =\int_{I} \operatorname{Pr}_{\nu_{i}, \mathcal{D}_{i}}^{0}(\Pi) \mu_{\nu, \mathcal{D}}(d i)
\end{aligned}
$$

For the induction step $(n>1)$, let $I \times \Pi \times M$ be a measurable rectangle in $\mathfrak{F}_{\text {Paths }^{n+1}}$ such that $I \in \mathfrak{F}_{\mathcal{S}} \times \mathfrak{F}_{\text {Act }} \times \mathfrak{B}\left(\mathbb{R}_{\geq 0}\right)$ is a set of initial triples, $\Pi \in \mathfrak{F}_{\text {Paths }^{n-1}}$ and $M \in \mathfrak{F}$ is a set of combined transitions. Using the induction hypothesis $\operatorname{Pr}_{\nu, \mathcal{D}}^{n}(I \times \Pi)=\int_{I} \operatorname{Pr}_{\nu_{i}, \mathcal{D}_{i}}^{n-1}(\Pi) \mu_{\nu, \mathcal{D}}(d i)$ we derive:

$$
\begin{array}{crl}
\operatorname{Pr}_{\nu, \mathcal{D}}^{n+1}(I \times \Pi \times M)=\int_{I \times \Pi} \mu_{\mathcal{D}}(\pi, M) \operatorname{Pr}_{\nu, \mathcal{D}}^{n}(d \pi) & \left(* \text { Definition } 4^{*}\right) \\
\quad=\int_{I \times \Pi} \mu_{\mathcal{D}}\left(i \circ \pi^{\prime}, M\right) \operatorname{Pr}_{\nu, \mathcal{D}}^{n}\left(d\left(i \circ \pi^{\prime}\right)\right) & \left(* \pi \simeq i \circ \pi^{\prime}\right)
\end{array}
$$




$$
\begin{aligned}
& =\int_{I} \int_{\Pi} \mu_{\mathcal{D}}\left(i \circ \pi^{\prime}, M\right) \operatorname{Pr}_{\nu_{i}, \mathcal{D}_{i}}^{n-1}\left(d \pi^{\prime}\right) \mu_{\nu, \mathcal{D}}(d i) \quad(* \text { ind. hypothesis * }) \\
& \left.=\int_{I} \int_{\Pi} \mu_{\mathcal{D}_{i}}\left(\pi^{\prime}, M\right) \operatorname{Pr}_{\nu_{i}, \mathcal{D}_{i}}^{n-1}\left(d \pi^{\prime}\right) \mu_{\nu, \mathcal{D}}(d i) \quad \quad \quad \text { ( definition of } \mathcal{D}_{i} *\right) \\
& =\int_{I} \operatorname{Pr}_{\nu_{i}, \mathcal{D}_{i}}^{n}(\Pi \times M) \mu_{\nu, \mathcal{D}}(d i) . \quad \quad \quad(* \text { Definition } 4 *)
\end{aligned}
$$

A class of pathological paths that are not ruled out by Def. 2 are infinite paths whose duration converges to some real constant, i.e. paths that visit infinitely many states in a finite amount of time. For $n=0,1,2, \ldots$, an increasing sequence $r_{n} \in \mathbb{R}_{\geq 0}$ is Zeno if it converges to a positive real number. For example, $r_{n}:=$ $\sum_{i=1}^{n} \frac{1}{2^{n}}$ converges to 1 , hence is Zeno.

Lemma 4. Let $k \in \mathbb{N}$ and $B=\mathcal{S} \times \Omega^{k} \times(A c t \times[0,1] \times \mathcal{S})^{\omega}$; then $\operatorname{Pr}_{\nu, \mathcal{D}}^{\omega}(B)=0$.

Proof. The proof goes along the lines of [5, Prop. 1]:

As $\mathcal{S}$ is finite, we can define $\Lambda:=\max \{E(s, \alpha) \mid s \in \mathcal{S}, \alpha \in A c t\}$. For $n \geq 0$, let $B^{n}:=\mathcal{S} \times \Omega^{k} \times(\text { Act } \times[0,1] \times \mathcal{S})^{n}$ be a measurable base and $B_{n}$ the induced infinite measurable rectangle. By induction on $n$, we show that $\operatorname{Pr}_{\nu, \mathcal{D}}^{\omega}\left(B_{n}\right) \leq$ $\left(1-e^{-\Lambda}\right)^{n}$ :

- Let $n=0$. Then $\operatorname{Pr}_{\nu, \mathcal{D}}^{\omega}\left(B_{0}\right)=\operatorname{Pr}_{\nu, \mathcal{D}}^{k}\left(\mathcal{S} \times \Omega^{k}\right)=1$.

- As induction hypothesis let $\operatorname{Pr}_{\nu, \mathcal{D}}^{\omega}\left(B_{n}\right) \leq\left(1-e^{-\Lambda}\right)^{n}$. For $B_{n+1}$ we obtain:

$$
\begin{aligned}
\operatorname{Pr}_{\nu, \mathcal{D}}^{\omega}\left(B_{n+1}\right)=\operatorname{Pr}_{\nu, \mathcal{D}}^{n+k+1}\left(B^{n} \times A c t \times[0,1] \times \mathcal{S}\right) \\
\quad=\int_{B^{n}} \mu_{\mathcal{D}}(\pi, A c t \times[0,1] \times \mathcal{S}) \operatorname{Pr}_{\nu, \mathcal{D}}^{n+k}(d \pi) \\
\quad=\int_{B^{n}}\left(\sum_{\alpha \in A c t} \mathcal{D}(\pi,\{\alpha\}) \cdot P(\pi \downarrow, \alpha, \mathcal{S}) \cdot \int_{[0,1]} E(\pi \downarrow, \alpha) e^{-E(\pi \downarrow, \alpha) t} d t\right) \operatorname{Pr}_{\nu, \mathcal{D}}^{n+k}(d \pi) \\
\quad=\int_{B^{n}} \sum_{\alpha \in A c t} \mathcal{D}(\pi,\{\alpha\}) \cdot P(\pi \downarrow, \alpha, \mathcal{S}) \cdot\left(1-e^{-E(\pi \downarrow, \alpha)}\right) \operatorname{Pr}_{\nu, \mathcal{D}}^{n+k}(d \pi) \\
\leq\left(1-e^{-\Lambda}\right) \cdot \int_{B^{n}} \underbrace{\sum_{\alpha \in A c t} \mathcal{D}(\pi,\{\alpha\}) \cdot P(\pi \downarrow, \alpha, \mathcal{S})}_{\leq 1} \operatorname{Pr}_{\nu, \mathcal{D}}^{n+k}(d \pi) \\
\quad \leq\left(1-e^{-\Lambda}\right) \cdot \int_{B^{n}} \operatorname{Pr}_{\nu, \mathcal{D}}^{n+k}(d \pi)=\left(1-e^{-\Lambda}\right) \cdot \operatorname{Pr}_{\nu, \mathcal{D}}^{n+k}\left(B^{n}\right) \\
\quad=\left(1-e^{-\Lambda}\right) \cdot \operatorname{Pr}_{\nu, \mathcal{D}}^{\omega}\left(B_{n}\right) \leq\left(1-e^{-\Lambda}\right)^{n+1} .
\end{aligned}
$$

Now $B_{0} \supseteq B_{1} \supseteq \cdots$ and the $B_{n}$ converge to $B$, i.e. $B_{n} \downarrow B$; hence $\operatorname{Pr}_{\nu, \mathcal{D}}^{\omega}\left(B_{n}\right) \rightarrow$ $\operatorname{Pr}_{\nu, \mathcal{D}}^{\omega}(B)$ by $[2,1.2 .7]$. Further $\lim _{n \rightarrow \infty} \operatorname{Pr}_{\nu, \mathcal{D}}^{\omega}\left(B_{n}\right)=0$ for $\operatorname{Pr}_{\nu, \mathcal{D}}^{\omega}$ is a measure (i.e. nonnegative) and $\lim _{n \rightarrow \infty}\left(1-e^{-\Lambda}\right)^{n}=0$. Thus $\operatorname{Pr}_{\nu, \mathcal{D}}^{\omega}(B)=0$.

With this result we can prove the following theorem which justifies to generally rule out Zeno behaviour:

Theorem 2 (Converging paths theorem). The probability measure of the set of converging paths is zero. 
Proof. Let ConvPaths $:=\left\{s_{0} \stackrel{\alpha_{0}, t_{0}}{\longrightarrow} s_{1} \stackrel{\alpha_{1}, t_{1}}{\longrightarrow} \cdots \mid \sum_{i=0}^{n} t_{i}\right.$ converges $\}$. For $\pi \in$ ConvPaths, the sequence $\sum_{i=0}^{\infty} t_{i}$ converges; thus $t_{i}$ converges to 0 and there exists $k \in \mathbb{N}$ such that $t_{i} \leq 1$ for all $i \geq k$. Hence ConvPaths $\subseteq \bigcup_{k=0}^{\infty} \mathcal{S} \times \Omega^{k} \times$ $(\text { Act } \times[0,1] \times \mathcal{S})^{\omega}$. By Lemma $4, \operatorname{Pr}_{\nu, \mathcal{D}}^{\omega}\left(\mathcal{S} \times \Omega^{k} \times(A c t \times[0,1] \times \mathcal{S})^{\omega}\right)=0$ for all $k \in \mathbb{N}$. Thus we obtain

$$
\begin{aligned}
\operatorname{Pr}_{\nu, \mathcal{D}}^{\omega}\left(\bigcup_{k=0}^{\infty} \mathcal{S} \times \Omega^{k} \times(\text { Act } \times\right. & {\left.[0,1] \times \mathcal{S})^{\omega}\right) } \\
& \leq \sum_{k=0}^{\infty} \operatorname{Pr}_{\nu, \mathcal{D}}^{\omega}\left(\mathcal{S} \times \Omega^{k} \times(\text { Act } \times[0,1] \times \mathcal{S})^{\omega}\right)=0
\end{aligned}
$$

But then ConvPaths is a subset of a set of measure zero; hence, on $\mathfrak{F}_{\text {Paths }}{ }^{\omega}$ completed ${ }^{6}$ w.r.t. $\operatorname{Pr}_{\nu, \mathcal{D}}^{\omega}$ we obtain $\operatorname{Pr}_{\nu, \mathcal{D}}^{\omega}($ ConvPaths $)=0$.

\section{Strong bisimilarity}

Strong bisimilarity $[8,23]$ is an equivalence on the set of states of a CTMDP which relates two states if they are equally labelled and exhibit the same stepwise behaviour. As shown in Theorem 6, strong bisimilarity allows one to aggregate the state space while preserving transient and long run measures.

In the following we denote the equivalence class of $s$ under equivalence $\mathcal{R} \subseteq$ $\mathcal{S} \times \mathcal{S}$ by $[s]_{\mathcal{R}}=\left\{s^{\prime} \in \mathcal{S} \mid\left(s, s^{\prime}\right) \in \mathcal{R}\right\} ;$ if $\mathcal{R}$ is clear from the context we also write $[s]$. Further, $\mathcal{S}_{\mathcal{R}}:=\left\{[s]_{\mathcal{R}} \mid s \in \mathcal{S}\right\}$ is the quotient space of $\mathcal{S}$ under $\mathcal{R}$.

Definition 6 (Strong bisimulation relation). Let $\mathcal{C}=(\mathcal{S}$, Act, $\mathbf{R}, A P, L)$ be a CTMDP. An equivalence $\mathcal{R} \subseteq \mathcal{S} \times \mathcal{S}$ is a strong bisimulation relation if $L(u)=L(v)$ for all $(u, v) \in \mathcal{R}$ and $\mathbf{R}(u, \alpha, C)=\mathbf{R}(v, \alpha, C)$ for all $\alpha \in$ Act and all $C \in \mathcal{S}_{\mathcal{R}}$.

Two states $u$ and $v$ are strongly bisimilar $(u \sim v)$ if there exists a strong bisimulation relation $\mathcal{R}$ such that $(u, v) \in \mathcal{R}$. Strong bisimilarity is the union of all strong bisimulation relations.

\section{Theorem 3 (Strong bisimilarity). Strong bisimilarity is}

1. an equivalence,

2. a strong bisimulation relation and

3. the largest strong bisimulation relation.

Proof. Let $\sim=\bigcup\{\mathcal{R} \mid \mathcal{R}$ is a strong bisimulation relation on $\mathcal{S}\}$ denote strong bisimilarity.

1. $\sim$ is an equivalence:

Reflexivity and symmetry follow directly from the definition.

We show transitivity: $(u, v) \in \sim$ and $(v, w) \in \sim \Longrightarrow(u, w) \in \sim$.

$$
\begin{aligned}
& (u, v) \in \sim \Longrightarrow \text { ex. strong bisimulation relation } \mathcal{R}_{1} \subseteq \sim \text { s.t. }(u, v) \in \mathcal{R}_{1} \\
& (v, w) \in \sim \Longrightarrow \text { ex. strong bisimulation relation } \mathcal{R}_{2} \subseteq \sim \text { s.t. }(v, w) \in \mathcal{R}_{2}
\end{aligned}
$$

\footnotetext{
${ }^{6}$ We may assume $\mathfrak{F}_{\text {Paths }} \omega$ to be complete, see [2, p. $\left.18 \mathrm{ff}\right]$.
} 


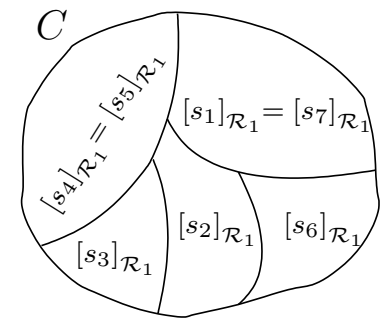

(a) according to $\mathcal{R}_{1}$

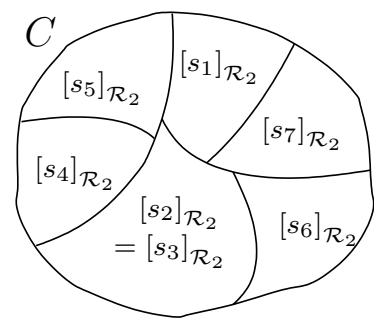

(b) according to $\mathcal{R}_{2}$

Fig. 2. Example partitioning of an equivalence class $C \in \mathcal{S}_{\mathcal{R}}$.

Let $\mathcal{R}$ denote the transitive closure of $\mathcal{R}_{1} \cup \mathcal{R}_{2}$. Then $(u, w) \in \mathcal{R}$. Therefore it suffices to show that $\mathcal{R}$ is a strong bisimulation relation. As $\mathcal{R}$ obviously is an equivalence, it remains to show that for all $(u, v) \in \mathcal{R}, \alpha \in$ Act and $C \in \mathcal{S}_{\mathcal{R}}$ it holds $L(u)=L(v)$ and

$$
\mathbf{R}(u, \alpha, C)=\mathbf{R}(v, \alpha, C) .
$$

The first condition, $L(u)=L(v)$ follows directly from the transitivity of the identity relation on $2^{A P}$. For condition (2), let $C=\left\{s_{1}, \ldots, s_{n}\right\}$. We have $C=\bigcup_{i=1}^{n}\left[s_{i}\right]_{\mathcal{R}_{k}}$ for $k \in\{1,2\}$ :

$\subseteq$ : Let $s \in C$. Then $s \in\left[s_{i}\right]_{\mathcal{R}_{k}}$ for some $i \in\{1, \ldots, n\}$. Hence $s \in \bigcup_{i=1}^{n}\left[s_{i}\right]_{\mathcal{R}_{k}}$. $\supseteq$ : Let $i \in\{1, \ldots, n\}$. Then it holds:

$$
\begin{aligned}
s \in\left[s_{i}\right]_{\mathcal{R}_{k}} & \Longleftrightarrow\left(s, s_{i}\right) \in \mathcal{R}_{k} & & \left(* \text { by definition }{ }^{*}\right) \\
& \Longleftrightarrow\left(s, s_{i}\right) \in \mathcal{R} & & \left(* \mathcal{R}_{k} \subseteq \mathcal{R}{ }^{*}\right) \\
& \Longleftrightarrow s \in\left[s_{i}\right]_{\mathcal{R}} & & \left(* \mathcal{R} \text { is an equivalence relation }{ }^{*}\right) \\
& \Longleftrightarrow s \in C & & \left(*\left[s_{i}\right]_{\mathcal{R}}=C^{*}\right)
\end{aligned}
$$

Hence we can decompose $C$ into equivalence classes w.r.t. $\mathcal{R}_{1}$ and $\mathcal{R}_{2}$ (see Fig. 2). As $\mathcal{R}_{1}$ is an equivalence relation, it induces a partitioning of $C$ :

$$
C=\biguplus\left\{\left[s_{i_{1}}\right]_{\mathcal{R}_{1}},\left[s_{i_{2}}\right]_{\mathcal{R}_{1}}, \ldots,\left[s_{i_{m}}\right]_{\mathcal{R}_{1}}\right\} \text { where } m \leq n .
$$

Note that the same applies to $\mathcal{R}_{2}$ for a different set of indices $i_{1}^{\prime}, \ldots, i_{m^{\prime}}^{\prime}$. Now we are able to prove property (2) by induction on the structure of $\mathcal{R}$. Therefore we provide an inductive definition of $\mathcal{R}$ as follows:

$$
\begin{aligned}
\mathcal{R}^{0} & =\mathcal{R}_{1} \cup \mathcal{R}_{2} \quad \text { and } \\
\mathcal{R}^{i+1} & =\left\{(u, w) \mid \exists v \in \mathcal{S} .(u, v) \in \mathcal{R}^{i} \wedge(v, w) \in \mathcal{R}^{i}\right\} \quad \text { for } i \geq 0 .
\end{aligned}
$$

By construction, the subset-ordering on $\mathcal{R}^{i}$ is bounded from above by $\mathcal{S} \times \mathcal{S}$. Further, $\mathcal{S}$ is finite, so that $\mathcal{R}^{0} \subseteq \mathcal{R}^{1} \subseteq \cdots$ is an ascending chain, that is, the transitive closure is reached after a finite number $z$ of iterations such that $\mathcal{R}^{z+1}=\mathcal{R}^{z}$. Obviously, we have $\mathcal{R}=\mathcal{R}^{z}$.

By induction on $i$, we prove that if $(u, v) \in \mathcal{R}^{i}$, then $\mathbf{R}(u, \alpha, C)=\mathbf{R}(v, \alpha, C)$ for all $\alpha \in$ Act and $C \in \mathcal{S}_{\mathcal{R}}$ :

- induction base $(i=0)$ :

Distinguish two cases: 
(a) Case 1: Let $(u, v) \in \mathcal{R}_{1}$ :

$$
\begin{aligned}
&(u, v) \in \mathcal{R}_{1} \Longrightarrow \forall C^{\prime} \in \mathcal{S}_{\mathcal{R}_{1}} \cdot \forall \alpha \in A c t . \mathbf{R}\left(u, \alpha, C^{\prime}\right)=\mathbf{R}\left(v, \alpha, C^{\prime}\right) \\
& \Longrightarrow \forall j \in\{1, \ldots, m\} . \forall \alpha \in \text { Act. } \\
& \mathbf{R}\left(u, \alpha,\left[s_{i_{j}}\right]_{\mathcal{R}_{1}}\right)=\mathbf{R}\left(v, \alpha,\left[s_{i_{j}}\right]_{\mathcal{R}_{1}}\right) \\
& \Longrightarrow \forall \alpha \in \text { Act. } \sum_{j=1}^{m} \mathbf{R}\left(u, \alpha,\left[s_{i_{j}}\right]_{\mathcal{R}_{1}}\right)=\sum_{j=1}^{m} \mathbf{R}\left(v, \alpha,\left[s_{i_{j}}\right]_{\mathcal{R}_{1}}\right) \\
& \Longrightarrow \forall \alpha \in \text { Act. } \mathbf{R}\left(u, \alpha, \biguplus_{j=1}^{m}\left[s_{i_{j}}\right]_{\mathcal{R}_{1}}\right)=\mathbf{R}\left(v, \alpha, \biguplus_{j=1}^{m}\left[s_{i_{j}}\right]_{\mathcal{R}_{1}}\right) \\
& \stackrel{(3)}{\Longrightarrow} \forall \alpha \in \text { Act. } \mathbf{R}(u, \alpha, C)=\mathbf{R}(v, \alpha, C) .
\end{aligned}
$$

(b) Case 2: Let $(u, v) \in \mathcal{R}_{2}$ :

The argument is completely analogue to the first case.

- induction step $(i \sim i+1)$ :

Assume $(u, w) \in \mathcal{R}^{i+1}$. By construction, we have $(u, v) \in \mathcal{R}^{i}$ and $(v, w) \in$ $\mathcal{R}^{i}$. Applying the induction hypothesis we have $\mathbf{R}(u, \alpha, C)=\mathbf{R}(v, \alpha, C)$ and $\mathbf{R}(v, \alpha, C)=\mathbf{R}(w, \alpha, C)$ for all actions $\alpha \in$ Act and all $C \in \mathcal{S}_{\mathcal{R}}$. Therefore $\mathbf{R}(u, \alpha, C)=\mathbf{R}(w, \alpha, C)$ directly follows from the transitivity of $=$ on $\mathbb{R}_{\geq 0}$.

Now we can conclude that $\sim$ is indeed transitive: Given $(u, v) \in \mathcal{R}_{1}$ and $(v, w) \in \mathcal{R}_{2}$, there exists a strong bisimulation relation $\mathcal{R}$ such that $(u, w) \in$ $\mathcal{R}$. By definition, $\mathcal{R} \subseteq \sim$; whence $u \sim w$.

2. $\sim$ is a strong bisimulation relation:

It remains to show for any $u \sim v$, that $\mathbf{R}(u, \alpha, C)=\mathbf{R}(v, \alpha, C)$ holds for all $\alpha \in A c t, C \in \tilde{\mathcal{S}}$. Since $u \sim v$ implies the existence of a strong bisimulation relation $\mathcal{R} \subseteq \sim$ with $(u, v) \in \mathcal{R}$ we may follow the idea of (3) to express $C$ as finite union of equivalence classes of $\mathcal{S}_{\mathcal{R}}$. Since $\mathcal{R}$ is a strong bisimulation relation, the rates from $u$ and $v$ into those equivalence classes are equal and maintained by summation.

3. $\sim$ is the largest (i.e. the coarsest) strong bisimulation relation:

Clear from the fact that $\sim$ is the union of all strong bisimulation relations.

Definition 7 (Quotient). Let $\mathcal{C}=(\mathcal{S}, A c t, \mathbf{R}, A P, L)$ be a CTMDP. Then $\tilde{\mathcal{C}}:=$ $(\tilde{\mathcal{S}}$, Act $, \tilde{\mathbf{R}}, A P, \tilde{L})$ where $\tilde{\mathcal{S}}:=\mathcal{S}_{\sim}, \tilde{\mathbf{R}}([s], \alpha, C):=\mathbf{R}(s, \alpha, C)$ and $\tilde{L}([s]):=L(s)$ for all $s \in \mathcal{S}, \alpha \in$ Act and $C \in \tilde{\mathcal{S}}$ is the quotient of $\mathcal{C}$ under strong bisimilarity.

For states $[s],[t] \in \tilde{\mathcal{S}}$ of the quotient $\tilde{\mathcal{C}}$, let $\tilde{E}([s], \alpha):=\sum_{s^{\prime} \in[s]} E(s, \alpha)$ be the exit rate of $[s]$ under action $\alpha$. Further, $\tilde{\mathbf{P}}([s], \alpha,[t]):=\frac{\tilde{\mathbf{R}}([s], \alpha,[t])}{\tilde{E}([s], \alpha)}$ is the discrete branching probability from state $[s]$ to state $[t]$ under action $\alpha$.

Example 3. Consider the CTMDP over the set $A P=\{a\}$ of atomic propositions in Fig. 3(a). Its quotient under strong bisimilarity is outlined in Fig. 3(b).

In the quotient, exit rates and branching probabilities are preserved w.r.t. the underlying CTMDP as shown by the following two lemmas: 


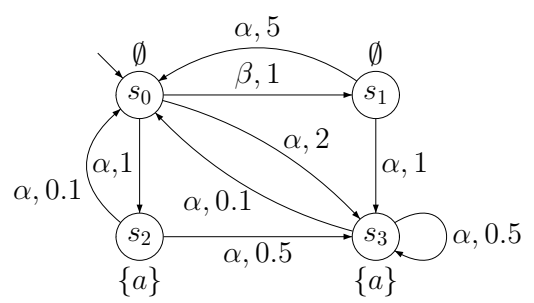

(a) $\operatorname{CTMDP} \mathcal{C}$

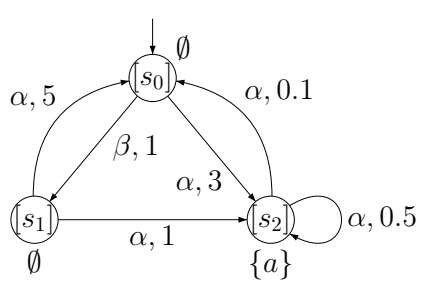

(b) Quotient $\tilde{\mathcal{C}}$

Fig. 3. Quotient under strong bisimilarity.

Lemma 5 (Preservation of exit rates). Let $\mathcal{C}=(\mathcal{S}$, Act, $\mathbf{R}, A P, L)$ be a $C T$ $M D P$ and $\tilde{\mathcal{C}}$ its quotient under strong bisimilarity. Then $E(s, \alpha)=\tilde{E}([s], \alpha)$ for all $s \in \mathcal{S}$ and $\alpha \in$ Act.

Proof. Let $\mathcal{S}=\bigcup_{k=0}^{n}\left[s_{i_{k}}\right]$ such that $\left[s_{i_{j}}\right] \cap\left[s_{i_{k}}\right]=\emptyset$ for all $j \neq k$. For all states $s \in \mathcal{S}$ it holds:

$$
\begin{aligned}
E(s, \alpha)=\sum_{s^{\prime} \in \mathcal{S}} \mathbf{R}\left(s, \alpha, s^{\prime}\right) & =\sum_{k=0}^{n} \sum_{s^{\prime} \in\left[s_{i_{k}}\right]} \mathbf{R}\left(s, \alpha, s^{\prime}\right)=\sum_{k=0}^{n} \mathbf{R}\left(s, \alpha,\left[s_{i_{k}}\right]\right) \\
& \stackrel{\text { Def. } 7}{=} \sum_{k=0}^{n} \tilde{\mathbf{R}}\left([s], \alpha,\left[s_{i_{k}}\right]\right)=\sum_{\left[s^{\prime}\right] \in \tilde{\mathcal{S}}} \tilde{\mathbf{R}}\left([s], \alpha,\left[s^{\prime}\right]\right)=\tilde{E}([s], \alpha) .
\end{aligned}
$$

With Lemma 5 it easily follows that the discrete transition probabilities are preserved under strong bisimulation:

Lemma 6 (Preservation of transition probabilities). Let $\mathcal{C}$ be as before and let $\tilde{\mathcal{C}}$ be its quotient under strong bisimilarity. For all states $s, t \in \mathcal{S}$ and all actions $\alpha \in$ Act it holds

$$
\tilde{\mathbf{P}}([s], \alpha,[t])=\sum_{t^{\prime} \in[t]} \mathbf{P}\left(s, \alpha, t^{\prime}\right)
$$

Proof.

$$
\begin{aligned}
\tilde{\mathbf{P}}([s], \alpha,[t]) & =\frac{\tilde{\mathbf{R}}([s], \alpha,[t])}{\tilde{E}([s], \alpha)} \stackrel{\text { Def. }}{=} \frac{\mathbf{R}(s, \alpha,[t])}{\tilde{E}([s], \alpha)} \\
& =\frac{\sum_{t^{\prime} \in[t]} \mathbf{R}\left(s, \alpha, t^{\prime}\right)}{\tilde{E}([s], \alpha)} \stackrel{\text { Lemma }}{=} \frac{\sum_{t^{\prime} \in[t]} \mathbf{R}\left(s, \alpha, t^{\prime}\right)}{E(s, \alpha)}=\sum_{t^{\prime} \in[t]} \mathbf{P}\left(s, \alpha, t^{\prime}\right) .
\end{aligned}
$$

\section{Continuous Stochastic Logic}

Continuous stochastic logic $[3,5]$ is a state-based logic to reason about continuoustime Markov chains. In this context, its formulas characterize strong bisimilarity [16] as defined in [5]; moreover, strongly bisimilar states satisfy the same CSL formulas [5]. In this paper, we extend CSL to CTMDPs along the lines of [6] and further introduce a long-run average operator [15]. Our semantics is based on ideas from $[9,11]$ where variants of PCTL are extended to (discrete time) MDPs. 


\subsection{Syntax and Semantics}

Definition 8 (CSL syntax). For $a \in A P, p \in[0,1], I \subseteq \mathbb{R}_{\geq 0}$ a nonempty interval and $\sqsubseteq \in\{<, \leq, \geq,>\}$, CSL state and CSL path formulas are defined by

$$
\Phi::=a|\neg \Phi| \Phi \wedge \Phi|\forall \sqsubseteq p \varphi|\left\llcorner\sqsubseteq p \Phi \quad \text { and } \quad \varphi::=\mathrm{X}^{I} \Phi \mid \Phi \bigcup^{I} \Phi .\right.
$$

The Boolean connectives $\vee$ and $\rightarrow$ are defined as usual; further we extend the syntax by deriving the timed modal operators "eventually" and "always" using the equalities $\diamond^{I} \Phi \equiv \operatorname{ttU}^{I} \Phi$ and $\square^{I} \Phi \equiv \neg \diamond^{I} \neg \Phi$ where tt $:=a \vee \neg a$ for some $a \in A P$. Similarly, the equality $\exists \sqsubseteq p \varphi \equiv \neg \forall \sqsupset p \varphi$ defines an existentially quantified transient state operator.

Example 4. Reconsider the CTMDP from Fig. 3(a). The transient state formula $\forall>0.1 \diamond^{[0,1]} a$ states that the probability to reach an $a$-labelled state within at most one time unit exceeds 0.1 no matter how the nondeterministic choices in the current state are resolved. Further, the long-run average formula $\mathrm{L}^{<0.25} \neg a$ states that for all scheduling decisions, the system spends less than $25 \%$ of its execution time in non- $a$ states, on average.

Formally the long-run average is derived as follows: For $B \subseteq \mathcal{S}$, let $\mathbf{I}_{B}$ denote an indicator with $\mathbf{I}_{B}(s)=1$ if $s \in B$ and 0 otherwise. Following the ideas of [15,24], we compute the fraction of time spent in states from the set $B$ on an infinite path $\pi$ up to time bound $t \in \mathbb{R}_{\geq 0}$ and define $\operatorname{avg}_{B, t}(\pi)=\frac{1}{t} \int_{0}^{t} \mathbf{I}_{B}\left(\pi @ t^{\prime}\right) d t^{\prime}$. As $\operatorname{avg}_{B, t}$ is a random variable, its expectation can be derived given an initial distribution $\nu \in \operatorname{Distr}(\mathcal{S})$ and a measurable scheduler $\mathcal{D} \in T H R$, i.e. $E\left(\operatorname{avg}_{B, t}\right)=$ $\int_{\text {Paths }}{ }^{a v g_{B, t}}(\pi) \operatorname{Pr}_{\nu, \mathcal{D}}^{\omega}(d \pi)$. Having the expectation for fixed time bound $t$, we now let $t \rightarrow \infty$ and obtain the long-run average as $\lim _{t \rightarrow \infty} E\left(a v g_{B, t}\right)$.

Definition 9 (CSL semantics). Let $\mathcal{C}=(\mathcal{S}$, Act, $\mathbf{R}, A P, L)$ be a CTMDP, $s, t \in \mathcal{S}, a \in A P, \sqsubseteq \in\{<, \leq, \geq\rangle$,$\} and \pi \in$ Paths $^{\omega}$. Further let $\nu_{s}(t):=1$ if $s=t$ and 0 otherwise. The semantics of state formulas is defined by

$$
\begin{aligned}
s \models a & \Longleftrightarrow a \in L(s) \\
s \models \neg \Phi & \Longleftrightarrow \text { not } s \models \Phi \\
s \models \Phi \wedge \Psi & \Longleftrightarrow s \models \Phi \text { and } s \models \Psi \\
s \models \forall \sqsubseteq p & \Longleftrightarrow \forall \mathcal{D} \in T H R . \quad \operatorname{Pr}_{\nu_{s}, \mathcal{D}}^{\omega}\left\{\pi \in \operatorname{Paths}^{\omega} \mid \pi \models \varphi\right\} \sqsubseteq p \\
s \models \mathrm{L} \sqsubseteq p & \Longleftrightarrow \forall \mathcal{D} \in T H R . \lim _{t \rightarrow \infty} \int_{\text {Paths }^{\omega}} \operatorname{avg}_{\text {Sat }(\Phi), t}(\pi) \operatorname{Pr}_{\nu_{s}, \mathcal{D}}^{\omega}(d \pi) \sqsubseteq p .
\end{aligned}
$$

Path formulas are defined by

$$
\begin{aligned}
& \pi \models \mathrm{X}^{I} \Phi \Longleftrightarrow \pi[1] \models \Phi \wedge \delta(\pi, 0) \in I \\
& \pi \models \Phi \mathrm{U}^{I} \Psi \Longleftrightarrow \exists t \in I .\left(\pi @ t \models \Psi \wedge\left(\forall t^{\prime} \in[0, t) . \pi @ t^{\prime} \models \Phi\right)\right)
\end{aligned}
$$

where $\operatorname{Sat}(\Phi):=\{s \in \mathcal{S} \mid s \models \Phi\}$ and $\delta(\pi, n)$ is the time spent in state $\pi[n]$.

In Def. 9 the transient-state operator $\forall \sqsubseteq p \varphi$ is based on the measure of the set of paths that satisfy $\varphi$. For this to be well defined we must show that the set $\left\{\pi \in\right.$ Paths $\left.^{\omega} \mid \pi \models \varphi\right\}$ is measurable:

Theorem 4 (Measurability of path formulas). The set $\left\{\pi \in\right.$ Paths $\left.^{\omega} \mid \pi \models \varphi\right\}$ is measurable for all CSL path formula $\varphi$. 


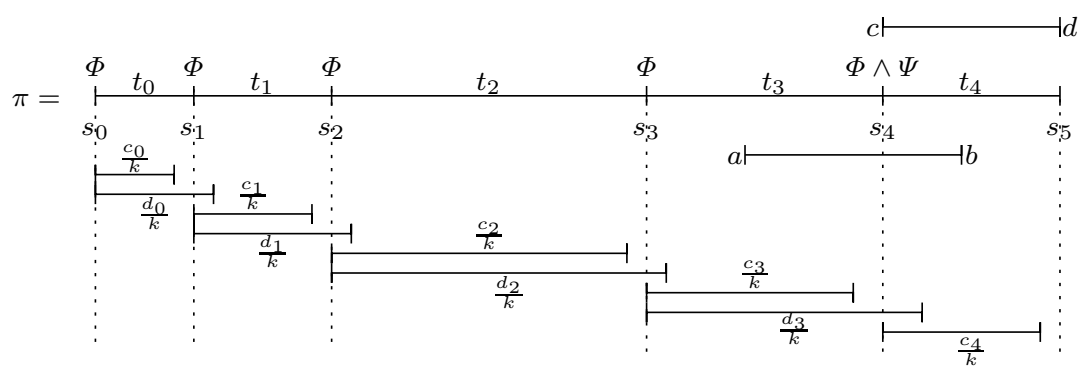

Fig. 4. Discretization of intervals with $n=4$ and $I=(a, b)$.

Proof. For next formulas, the proof is straightforward. For until formulas, let $\pi=s_{0} \stackrel{\alpha_{0}, t_{0}}{\longrightarrow} s_{1} \stackrel{\alpha_{1}, t_{1}}{\longrightarrow} \cdots \in$ Paths $^{\omega}$ and assume $\pi \models \Phi \bigcup^{I} \Psi$. By Def. 9 it holds $\pi \models \Phi \bigcup^{I} \Psi$ iff $\exists t \in I .\left(\pi @ t \models \Psi \wedge \forall t^{\prime} \in[0, t) . \pi @ t^{\prime} \models \Phi\right)$. As we may exclude Zeno behaviour by Theorem 2 , there exists $n \in \mathbb{N}$ with $\pi @ t=\pi[n]=s_{n}$ such that $I$ and the period of time $\left[\sum_{i=0}^{n-1} t_{i}, \sum_{i=0}^{n} t_{i}\right)$ spent in state $s_{n}$ overlap; further $s_{n} \models \Psi$ and $s_{i} \models \Phi$ for $i=0, \ldots, n-1$. Note however, that $s_{n}$ must also satisfy $\Phi$ except for the case of instantaneous arrival where $\sum_{i=0}^{n-1} t_{i} \in I$. Accordingly, the set $\left\{\pi \in\right.$ Paths $\left.^{\omega} \mid \pi \models \Phi \mathrm{U}^{I} \Psi\right\}$ can be represented by the union

$$
\begin{aligned}
& \bigcup_{n=0}^{\infty}\left\{\pi \in \text { Paths }^{\omega} \mid \sum_{i=0}^{n-1} t_{i} \in I \wedge \pi[n] \models \Psi \wedge \forall m<n . \pi[m] \models \Phi\right\} \\
& \cup \bigcup_{n=0}^{\infty}\left\{\pi \in \text { Paths }^{\omega} \mid\left(\sum_{i=0}^{n-1} t_{i}, \sum_{i=0}^{n} t_{i}\right) \cap I \neq \emptyset \wedge \pi[n] \models \Psi \wedge \forall m \leq n . \pi[m] \models \Phi\right\} .
\end{aligned}
$$

It suffices to show that the subsets of (4) and (5) induced by any $n \in \mathbb{N}$ are measurable cylinders. In the following, we exhibit the proof for (5) and closed intervals $I=[a, b]$ as the other cases are similar. For fixed $n \geq 0$ we show that the corresponding cylinder base is measurable using a discretization argument:

$$
\begin{aligned}
&\left\{\pi \in \text { Paths }^{n+1} \mid\left(\sum_{i=0}^{n-1} t_{i}, \sum_{i=0}^{n} t_{i}\right) \cap[a, b] \neq \emptyset \wedge \pi[n] \models \Psi \wedge \forall m \leq n . \pi[m] \models \Phi\right\} \\
&= \bigcup_{\substack{k=1 \\
d_{0}+\cdots+c_{n} \geq a k}}^{\infty} \prod_{i=0}^{n-1}\left[\operatorname{Sat}(\Phi) \times \operatorname{Act} \times\left(\frac{c_{i}}{k}, \frac{d_{i}}{k}\right)\right] \times \operatorname{Sat}(\Phi \wedge \Psi) \times \operatorname{Act} \times\left(\frac{c_{n}}{k}, \infty\right) \times \mathcal{S} \\
& c_{i}<d_{i}
\end{aligned}
$$

where $c_{i}, d_{j} \in \mathbb{N}$. To shorten notation, let $c:=\sum_{i=0}^{n-1} t_{i}$ and $d:=\sum_{i=0}^{n} t_{i}$.

$\subseteq$ : Let $\pi=s_{0} \stackrel{\alpha_{0}, t_{0}}{\longrightarrow} s_{1} \stackrel{\alpha_{1}, t_{1}}{\longrightarrow} \cdots \stackrel{\alpha_{n}, t_{n}}{\longrightarrow} s_{n+1}$ be in the set on the left-hand side of equation (6). The intervals $(c, d)$ and $[a, b]$ overlap, hence $c<b$ and $d>a$ (see top of Fig. 4). Further $\pi[i] \models \Phi$ for $i=0, \ldots, n$ and $\pi[n] \models \Psi$. To show that $\pi$ is in the set on the right-hand side, let $c_{i}=\left\lceil t_{i} \cdot k-1\right\rceil$ and $d_{i}=\left\lfloor t_{i} \cdot k+1\right\rfloor$ for $k>0$. Then $\frac{c_{i}}{k}<t_{i}<\frac{d_{i}}{k}$ approximates the sojourn times $t_{i}$ as depicted in Fig. 4. Further let $\varepsilon=\sum_{i=0}^{n} t_{i}-a$ and choose $k_{0}$ such that $\frac{n+1}{k_{0}} \leq \varepsilon$ to obtain

$$
a=\sum_{i=0}^{n} t_{i}-\varepsilon \leq \sum_{i=0}^{n} t_{i}-\frac{n+1}{k_{0}} \leq \sum_{i=0}^{n} \frac{c_{i}+1}{k_{0}}-\frac{n+1}{k_{0}}=\sum_{i=0}^{n} \frac{c_{i}}{k_{0}} .
$$




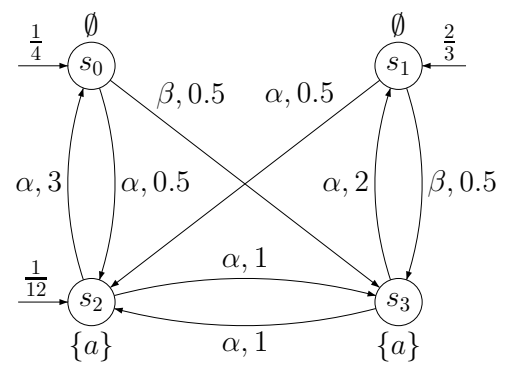

(a) CTMDP $\mathcal{C}$ and initial distr.

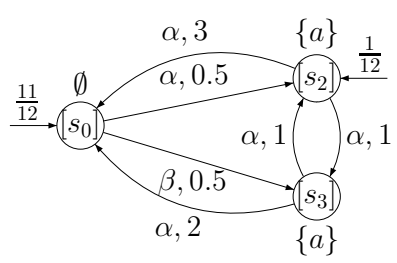

(b) Quotient $\tilde{\mathcal{C}}$

Fig. 5. Derivation of the quotient scheduler.

Thus $a k \leq \sum_{i=0}^{n} c_{i}$ for all $k \geq k_{0}$. Similarly, we obtain $k_{0}^{\prime} \in \mathbb{N}$ s.t. $\sum_{i=0}^{n-1} d_{i} \leq b k$ for all $k \geq k_{0}^{\prime}$. Hence for large $k, \pi$ is in the set on the right-hand side.

․: Let $\pi$ be in the set on the right-hand side of equation (6) with corresponding values for $c_{i}, d_{i}$ and $k$. Then $t_{i} \in\left(\frac{c_{i}}{k}, \frac{d_{i}}{k}\right)$. Hence $a \leq \sum_{i=0}^{n} \frac{c_{i}}{k}<\sum_{i=0}^{n} t_{i}=d$ and $b \geq \sum_{i=0}^{n-1} \frac{d_{i}}{k}>\sum_{i=0}^{n-1} t_{i}=c$ so that the time-interval $(c, d)$ of state $s_{n}$ and the time interval $I=[a, b]$ of the formula overlap. Further, $\pi[m] \models \Phi$ for $m \leq n$ and $\pi[n] \models \Psi$; thus $\pi$ is in the set on the left-hand side of equation (6).

The right-hand side of equation (6) is measurable, hence also the cylinder base. This extends to its cylinder and the countable union in equation (5).

\subsection{Strong bisimilarity preserves CSL}

We now prepare the main result of our paper. To prove that strong bisimilarity preserves CSL formulas we establish a correspondence between certain sets of paths of a CTMDP and its quotient which is measure-preserving:

Definition 10 (Simple bisimulation closed). Let $\mathcal{C}=(\mathcal{S}$, Act, $\mathbf{R}, A P, L)$ be a CTMDP. A measurable rectangle $\Pi=S_{0} \times A_{0} \times T_{0} \times \cdots \times A_{n-1} \times T_{n-1} \times S_{n}$ is simple bisimulation closed if $S_{i} \in(\tilde{\mathcal{S}} \cup\{\emptyset\})$ for $i=0, \ldots, n$. Further, let $\tilde{\Pi}=\left\{S_{0}\right\} \times A_{0} \underset{\tilde{C}}{\times} T_{0} \times \cdots \times A_{n-1} \times T_{n-1} \times\left\{S_{n}\right\}$ be the corresponding rectangle in the quotient $\tilde{\mathcal{C}}$.

An essential step in our proof strategy is to obtain a scheduler on the quotient. The following example illustrates the intuition for such a scheduler.

Example 5. Let $\mathcal{C}$ be the CTMDP in Fig. 5(a) where $\nu\left(s_{0}\right)=\frac{1}{4}, \nu\left(s_{1}\right)=\frac{2}{3}$ and $\nu\left(s_{2}\right)=\frac{1}{12}$. Assume a scheduler $\mathcal{D}$ where $\mathcal{D}\left(s_{0},\{\alpha\}\right)=\frac{2}{3}, \mathcal{D}\left(s_{0},\{\beta\}\right)=\frac{1}{3}$, $\mathcal{D}\left(s_{1},\{\alpha\}\right)=\frac{1}{4}$ and $\mathcal{D}\left(s_{1},\{\beta\}\right)=\frac{3}{4}$. Intuitively, a scheduler $\mathcal{D}_{\sim}^{\nu}$ that mimics $\mathcal{D}^{\prime}$ 's behaviour on the quotient $\tilde{\mathcal{C}}$ in Fig. 5 (b) can be defined by

$$
\begin{aligned}
& \mathcal{D}_{\sim}^{\nu}\left(\left[s_{0}\right],\{\alpha\}\right)=\frac{\sum_{s \in\left[s_{0}\right]} \nu(s) \cdot \mathcal{D}(s,\{\alpha\})}{\sum_{s \in\left[s_{0}\right]} \nu(s)}=\frac{\frac{1}{4} \cdot \frac{2}{3}+\frac{2}{3} \cdot \frac{1}{4}}{\frac{1}{4}+\frac{2}{3}}=\frac{4}{11} \quad \text { and } \\
& \mathcal{D}_{\sim}^{\nu}\left(\left[s_{0}\right],\{\beta\}\right)=\frac{\sum_{s \in\left[s_{0}\right]} \nu(s) \cdot \mathcal{D}(s,\{\beta\})}{\sum_{s \in\left[s_{0}\right]} \nu(s)}=\frac{\frac{1}{4} \cdot \frac{1}{3}+\frac{2}{3} \cdot \frac{3}{4}}{\frac{1}{4}+\frac{2}{3}}=\frac{7}{11} .
\end{aligned}
$$

Even though $s_{0}$ and $s_{1}$ are bisimilar, the scheduler $\mathcal{D}$ decides differently for the histories $\pi_{0}=s_{0}$ and $\pi_{1}=s_{1}$. As $\pi_{0}$ and $\pi_{1}$ collapse into $\tilde{\pi}=\left[s_{0}\right]$ on the quotient, $\mathcal{D}_{\sim}^{\nu}$ can no longer distinguish between $\pi_{0}$ and $\pi_{1}$. Therefore $\mathcal{D}$ 's decision for any history $\pi \in \tilde{\pi}$ is weighed w.r.t. the total probability of $\tilde{\pi}$. 
Definition 11 (Quotient scheduler). Let $\mathcal{C}=(\mathcal{S}, A c t, \mathbf{R}, A P, L)$ be a $C T$ $M D P, \nu \in \operatorname{Distr}(\mathcal{S})$ and $\mathcal{D} \in T H R$. First, define the history weight of finite paths of length $n$ inductively as follows:

$$
\begin{aligned}
h w_{0}\left(\nu, \mathcal{D}, s_{0}\right) & :=\nu\left(s_{0}\right) \text { and } \\
h w_{n+1}\left(\nu, \mathcal{D}, \pi \stackrel{\alpha_{n}, t_{n}}{\longrightarrow} s_{n+1}\right) & :=h w_{n}(\nu, \mathcal{D}, \pi) \cdot \mathcal{D}\left(\pi,\left\{\alpha_{n}\right\}\right) \cdot \mathbf{P}\left(\pi \downarrow, \alpha_{n}, s_{n+1}\right) .
\end{aligned}
$$

Let $\tilde{\pi}=\left[s_{0}\right] \stackrel{\alpha_{0}, t_{0}}{\longrightarrow} \cdots \stackrel{\alpha_{n-1}, t_{n-1}}{\longrightarrow}\left[s_{n}\right]$ be a timed history of $\tilde{\mathcal{C}}$ and $\Pi=\left[s_{0}\right] \times$ $\left\{\alpha_{0}\right\} \times\left\{t_{0}\right\} \times \cdots \times\left\{\alpha_{n-1}\right\} \times\left\{t_{n-1}\right\} \times\left[s_{n}\right]$ be the corresponding set of paths in $\mathcal{C}$. The quotient scheduler $\mathcal{D}_{\sim}^{\nu}$ on $\tilde{\mathcal{C}}$ is then defined as follows:

$$
\mathcal{D}_{\sim}^{\nu}\left(\tilde{\pi}, \alpha_{n}\right):=\frac{\sum_{\pi \in \Pi} h w_{n}(\nu, \mathcal{D}, \pi) \cdot \mathcal{D}\left(\pi,\left\{\alpha_{n}\right\}\right)}{\sum_{\pi \in \Pi} h w_{n}(\nu, \mathcal{D}, \pi)} .
$$

Further, let $\tilde{\nu}([s]):=\sum_{s^{\prime} \in[s]} \nu\left(s^{\prime}\right)$ be the initial distribution on $\tilde{\mathcal{C}}$.

A history $\tilde{\pi}$ of $\tilde{\mathcal{C}}$ corresponds to a set of paths $\Pi$ in $\mathcal{C}$; given $\tilde{\pi}$, the quotient scheduler decides by multiplying $\mathcal{D}$ 's decision on each path in $\Pi$ with its corresponding weight and normalizing with the weight of $\Pi$ afterwards. Now we obtain a first intermediate result: For CTMDP $\mathcal{C}$, if $\Pi$ is a simple bisimulation closed set of paths, $\nu$ an initial distribution and $\mathcal{D} \in T H R$, the measure of $\Pi$ in $\mathcal{C}$ coincides with the measure of $\tilde{\Pi}$ in $\tilde{\mathcal{C}}$ which is induced by $\tilde{\nu}$ and $\mathcal{D}_{\sim}^{\nu}$ :

Theorem 5. Let $\mathcal{C}$ be a $C T M D P$ with set of states $\mathcal{S}$ and $\nu \in \operatorname{Distr}(\mathcal{S})$. Then $\operatorname{Pr}_{\nu, \mathcal{D}}^{\omega}(\Pi)=\operatorname{Pr}_{\tilde{\nu}, \mathcal{D}_{\sim}^{\nu}}^{\omega}(\tilde{\Pi})$ where $\mathcal{D} \in T H R$ and $\Pi$ simple bisimulation closed.

Proof. By induction on the length $n$ of cylinder bases. The induction base holds for all $\nu \in \operatorname{Distr}(\mathcal{S})$ since $\operatorname{Pr}_{\nu, \mathcal{D}}^{0}([s])=\sum_{s^{\prime} \in[s]} \nu\left(s^{\prime}\right)=\tilde{\nu}([s])=\operatorname{Pr}_{\tilde{\nu}, \mathcal{D}_{\sim}^{\nu}}^{0}(\{[s]\})$. With the induction hypothesis that $\operatorname{Pr}_{\nu, \mathcal{D}}^{n}(\Pi)=\operatorname{Pr}_{\tilde{\nu}, \mathcal{D}_{\sim}^{\nu}}^{n}(\tilde{\Pi})$ for all $\nu \in \operatorname{Distr}(\mathcal{S})$, $\mathcal{D} \in T H R$ and bisimulation closed $\Pi \subseteq$ Paths $^{n}$ we obtain the induction step:

$$
\begin{aligned}
& \operatorname{Pr}_{\nu, \mathcal{D}}^{n+1}\left(\left[s_{0}\right] \times A_{0} \times T_{0} \times \Pi\right)=\int_{\left[s_{0}\right] \times A_{0} \times T_{0}} \operatorname{Pr}_{\mathbf{P}(s, \alpha, \cdot), \mathcal{D}(s \stackrel{\alpha, t}{\longrightarrow} \cdot)}(\Pi) \mu_{\nu, \mathcal{D}}(d s, d \alpha, d t) \\
& =\int_{s \in\left[s_{0}\right]} \nu(d s) \int_{\alpha \in A_{0}} \mathcal{D}(s, d \alpha) \int_{T_{0}} \operatorname{Pr}_{\mathbf{P}(s, \alpha, \cdot), \mathcal{D}(s \stackrel{\alpha, t}{\longrightarrow} .)}(\Pi) \eta_{E(s, \alpha)}(d t) \\
& \left.=\sum_{s \in\left[s_{0}\right]} \nu(s) \sum_{\alpha \in A_{0}} \mathcal{D}(s,\{\alpha\}) \int_{T_{0}} \operatorname{Pr}_{\mathbf{P}(s, \alpha, \cdot), \mathcal{D}(s \stackrel{\alpha, t}{\longrightarrow} \cdot)}(\Pi) \eta_{\tilde{E}\left(\left[s_{0}\right], \alpha\right)}(d t) \quad \text { (* by Lemma } 5^{*}\right) \\
& \stackrel{\text { i.h. }}{=} \sum_{s \in\left[s_{0}\right]} \sum_{\alpha \in A_{0}} \int_{T_{0}} \operatorname{Pr}_{\tilde{\mathbf{P}}\left(\left[s_{0}\right], \alpha, \cdot\right), \mathcal{D} \nu}^{n}\left(\left[s_{0}\right] \stackrel{\alpha, t}{\longrightarrow} \cdot\right)(\tilde{I}) \cdot \nu(s) \cdot \mathcal{D}(s,\{\alpha\}) \eta_{\tilde{E}\left(\left[s_{0}\right], \alpha\right)}(d t) \\
& =\sum_{\alpha \in A_{0}} \int_{T_{0}} \operatorname{Pr}_{\tilde{\mathbf{P}}\left(\left[s_{0}\right], \alpha, \cdot\right), \mathcal{D} \nu\left(\left[s_{0}\right] \stackrel{\alpha, t}{\longrightarrow} \cdot\right)}(\tilde{\Pi}) \cdot \sum_{s \in\left[s_{0}\right]}(\nu(s) \cdot \mathcal{D}(s,\{\alpha\})) \eta_{\tilde{E}\left(\left[s_{0}\right], \alpha\right)}(d t) \\
& =\sum_{\alpha \in A_{0}} \int_{T_{0}} \operatorname{Pr}_{\tilde{\mathbf{P}}\left(\left[s_{0}\right], \alpha, \cdot\right), \mathcal{D} \sim\left(\left[s_{0}\right] \stackrel{\alpha, t}{\longrightarrow} \cdot\right)}^{n}(\tilde{I}) \cdot\left(\sum_{s \in\left[s_{0}\right]} \nu(s)\right) \cdot \frac{\sum_{s \in\left[s_{0}\right]} \nu(s) \cdot \mathcal{D}(s,\{\alpha\})}{\sum_{s \in\left[s_{0}\right]} \nu(s)} \eta_{\tilde{E}\left(\left[s_{0}\right], \alpha\right)}(d t) \\
& =\sum_{\alpha \in A_{0}} \int_{T_{0}} \operatorname{Pr}_{\tilde{\mathbf{P}}\left(\left[s_{0}\right], \alpha, \cdot\right), \mathcal{D} \nu}^{\nu}\left(\left[s_{0}\right] \stackrel{\alpha, t}{\longrightarrow} .\right)(\tilde{I}) \cdot \tilde{\nu}\left(\left[s_{0}\right]\right) \cdot \mathcal{D}_{\sim}^{\nu}\left(\left[s_{0}\right],\{\alpha\}\right) \eta_{\tilde{E}\left(\left[s_{0}\right], \alpha\right)}(d t) \\
& =\int_{\left\{\left[s_{0}\right]\right\}} \tilde{\nu}(d[s]) \int_{A_{0}} \mathcal{D}_{\sim}^{\nu}([s], d \alpha) \int_{T_{0}} \operatorname{Pr}_{\tilde{\mathbf{P}}([s], \alpha, \cdot), \mathcal{D} \nu}^{n}([s] \stackrel{\alpha, t}{\longrightarrow} \cdot)(\tilde{\Pi}) \eta_{\tilde{E}([s], \alpha)}(d t) \\
& =\int_{\left\{\left[s_{0}\right]\right\} \times A_{0} \times T_{0}} \operatorname{Pr}_{\tilde{\mathbf{P}}([s], \alpha, \cdot), \mathcal{D}_{\sim}^{\nu}([s] \stackrel{\alpha, t}{\longrightarrow} \cdot)}^{n}(\tilde{I}) \tilde{\mu}_{\tilde{\nu}, \mathcal{D}_{\sim}^{\nu}}(d[s], d \alpha, d t)
\end{aligned}
$$




$$
=\operatorname{Pr}_{\tilde{\nu}, \mathcal{D} \nu}^{n+1}\left(\left\{\left[s_{0}\right]\right\} \times A_{0} \times T_{0} \times \tilde{\Pi}\right)
$$

where $\tilde{\mu}_{\tilde{\nu}, \mathcal{D}}^{\nu}$ is the extension of $\mu_{\nu, \mathcal{D}}$ (Def. 5 ) to sets of initial triples in $\tilde{\mathcal{C}}$ :

$$
\tilde{\mu}_{\tilde{\nu}, \mathcal{D}} \sim \mathfrak{F}_{\tilde{\mathcal{S}} \times A c t \times \mathbb{R}_{\geq 0} \rightarrow}[0,1]: I \mapsto \int_{\tilde{\mathcal{S}}}^{\tilde{\mathcal{S}}}(d[s]) \int_{A c t} \mathcal{D}_{\sim}^{\nu}([s], d \alpha) \int_{\mathbb{R}_{\geq 0}} \mathbf{I}_{I}([s], \alpha, t) \eta_{\tilde{E}([s], \alpha)}(d t) .
$$

According to Theorem 5, the quotient scheduler preserves the measure for simple bisimulation closed sets of paths, i.e. for paths, whose state components are equivalence classes under $\sim$. To generalize this to sets of paths that satisfy a CSL path formula, we introduce general bisimulation closed sets of paths:

Definition 12 (Bisimulation closed). Let $\mathcal{C}=(\mathcal{S}, A c t, \mathbf{R}, A P, L)$ be a $C T$ $M D P$ and $\tilde{\mathcal{C}}$ its quotient under strong bisimilarity. A measurable rectangle $\Pi=$ $S_{0} \times A_{0} \times T_{0} \times \cdots \times A_{n-1} \times T_{n-1} \times S_{n}$ is bisimulation closed if $S_{i}=\biguplus_{j=0}^{k_{i}}\left[s_{i, j}\right]$ for $k_{i} \in \mathbb{N}$ and $0 \leq i \leq n$. Let $\tilde{\Pi}=\bigcup_{j=0}^{k_{0}}\left\{\left[s_{0, j}\right]\right\} \times A_{0} \times T_{0} \times \cdots \times A_{n-1} \times T_{n-1} \times$ $\bigcup_{j=0}^{k_{n}}\left\{\left[s_{n, j}\right]\right\}$ be the corresponding rectangle in the quotient $\tilde{\mathcal{C}}$.

Lemma 7. Any bisimulation closed set of paths $\Pi$ can be represented as a finite disjoint union of simple bisimulation closed sets of paths.

Proof. Direct consequence of Def. 12.

Corollary 1. Let $\mathcal{C}$ be a $C T M D P$ with set of states $\mathcal{S}$ and $\nu \in \operatorname{Distr}(\mathcal{S})$ an initial distribution. Then $\operatorname{Pr}_{\nu, \mathcal{D}}^{\omega}(\Pi)=\operatorname{Pr}_{\tilde{\nu}, \mathcal{D}_{\sim}^{\nu}}^{\omega}(\tilde{\Pi})$ for any $\mathcal{D} \in T H R$ and any bisimulation closed set of paths $\Pi$.

Proof. Follows directly from Lemma 7 and Theorem 5.

Using these extensions we can now prove our main result:

Theorem 6. Let $\mathcal{C}$ be a CTMDP with set of states $\mathcal{S}$ and $u, v \in \mathcal{S}$. Then $u \sim v$ implies $u \models \Phi$ iff $v \models \Phi$ for all CSL state formulas $\Phi$.

Proof. By structural induction on $\Phi$. If $\Phi=a$ and $a \in A P$ the induction base follows as $L(u)=L(v)$. In the induction step, conjunction and negation are obvious.

Let $\Phi=\forall \sqsubseteq p \varphi$ and $\Pi=\left\{\pi \in\right.$ Paths $\left.^{\omega} \mid \pi \models \varphi\right\}$. To show $u \models \forall \sqsubseteq p \varphi$ implies $v \models \forall \sqsubseteq p \varphi$ it suffices to show that for any $\mathcal{V} \in T H R$ there exists $\mathcal{U} \in T H R$ with $\operatorname{Pr}_{\nu_{u}, \mathcal{U}}^{\omega}(\Pi)=\operatorname{Pr}_{\nu_{v}, \mathcal{V}}^{\omega}(\Pi)$. By Theorem 4 the set $\Pi$ is measurable, hence $\Pi=\biguplus_{i=0}^{\infty} \Pi_{i}$ for disjoint $\Pi_{i} \in \mathfrak{F}_{\text {Paths }} \omega$. By induction hypothesis for path formulas $\mathrm{X}^{I} \Phi$ and $\Phi \mathrm{U}^{I} \Psi$ the sets $\operatorname{Sat}(\Phi)$ and $\operatorname{Sat}(\Psi)$ are disjoint unions of $\sim$-equivalence classes. The same holds for any Boolean combination of $\Phi$ and $\Psi$. Hence $\Pi=$ $\biguplus_{i=0}^{\infty} \Pi_{i}$ where the $\Pi_{i}$ are bisimulation closed. For all $\mathcal{V} \in T H R$ and $\pi=s_{0} \stackrel{\alpha_{0}, t_{0}}{\longrightarrow}$ $\ldots \stackrel{\alpha_{n-1}, t_{n-1}}{\longrightarrow} s_{n}$ let $\mathcal{U}(\pi):=\mathcal{V}_{\sim}^{\nu_{v}}\left(\left[s_{0}\right] \stackrel{\alpha_{0}, t_{0}}{\longrightarrow} \cdots \stackrel{\alpha_{n-1}, t_{n-1}}{\longrightarrow}\left[s_{n}\right]\right)$. Thus $\mathcal{U}$ mimics on $\pi$ the decision of $\mathcal{V}_{\sim}^{\nu_{v}}$ on $\tilde{\pi}$. In fact $\mathcal{U}_{\sim}^{\nu_{u}}=\mathcal{V}_{\sim}^{\nu_{v}}$ since

$$
\mathcal{U}_{\sim}^{\nu_{u}}\left(\tilde{\pi}, \alpha_{n}\right)=\frac{\sum_{\pi \in \Pi} h w_{n}\left(\nu_{u}, \mathcal{U}, \pi\right) \cdot \mathcal{V}_{\sim}^{\nu_{v}}\left(\tilde{\pi}, \alpha_{n}\right)}{\sum_{\pi \in \Pi} h w_{n}\left(\nu_{u}, \mathcal{U}, \pi\right)}
$$


and $\mathcal{V}_{\sim}^{\nu_{v}}\left(\tilde{\pi}, \alpha_{n}\right)$ is independent of $\pi$. With $\tilde{\nu}_{u}=\tilde{\nu}_{v}$ and by Corollary 1 we obtain $\operatorname{Pr}_{\nu_{u}, \mathcal{U}}^{\omega}\left(\Pi_{i}\right)=\operatorname{Pr}_{\tilde{\nu}_{u}, \mathcal{U}_{\sim}^{\nu}}^{\omega}\left(\tilde{\Pi}_{i}\right)=\operatorname{Pr}_{\tilde{\nu}_{v}, \mathcal{V}_{\sim}^{\nu}}^{\omega}\left(\tilde{\Pi}_{i}\right)=\operatorname{Pr}_{\nu_{v}, \mathcal{V}}^{\omega}\left(\Pi_{i}\right)$ which carries over to $\Pi$ for $\Pi$ is a countable union of disjoint sets $\Pi_{i}$.

Let $\Phi=\mathrm{L} \sqsubseteq p \Psi$. Since $u \sim v$, it suffices to show that for all $s \in \mathcal{S}$ it holds $s \models \mathrm{L} \sqsubseteq p \Psi$ iff $[s] \models \mathrm{L} \sqsubseteq p \Psi$. The expectation of $\operatorname{avg}_{S a t(\Psi), t}$ for $t \in \mathbb{R}_{\geq 0}$ can be expressed as follows:

$$
\begin{aligned}
\int_{\text {Paths }}\left(\frac{1}{t} \int_{0}^{t} \mathbf{I}_{S a t(\Psi)}\left(\pi @ t^{\prime}\right) d t^{\prime}\right) P r_{\nu_{s}, \mathcal{D}}^{\omega} & (d \pi) \\
& =\frac{1}{t} \int_{0}^{t} \operatorname{Pr}_{\nu_{s}, \mathcal{D}}^{\omega}\left\{\pi \in P a t h s^{\omega} \mid \pi @ t^{\prime} \models \Psi\right\} d t^{\prime} .
\end{aligned}
$$

Further, the sets $\left\{\pi \in\right.$ Paths $\left.^{\omega} \mid \pi @ t^{\prime} \models \Psi\right\}$ and $\left\{\pi \in \operatorname{Paths}^{\omega} \mid \pi \models \diamond^{\left[t^{\prime}, t^{\prime}\right]} \Psi\right\}$ have the same measure and the induction hypothesis applies to $\Psi$. Applying the previous reasoning for the until case to the formula $t \mathrm{t} \mathrm{U}^{\left[t^{\prime}, t^{\prime}\right]} \Psi$ once, we obtain

$$
\operatorname{Pr}_{\nu_{s}, \mathcal{D}}^{\omega}\left\{\pi \in \operatorname{Paths}^{\omega}(\mathcal{C}) \mid \pi \models \diamond^{\left[t^{\prime}, t^{\prime}\right]} \Psi\right\}=\operatorname{Pr}_{\tilde{\nu}_{s}, \mathcal{D}_{\sim}^{\nu_{s}}}^{\omega}\left\{\tilde{\pi} \in \operatorname{Paths}^{\omega}(\tilde{\mathcal{C}}) \mid \tilde{\pi}=\diamond^{\left[t^{\prime}, t^{\prime}\right]} \Psi\right\}
$$

for all $t^{\prime} \in \mathbb{R}_{\geq 0}$. Thus the expectations of $\operatorname{avg}_{\text {Sat }(\Psi), t}$ on $\mathcal{C}$ and $\tilde{\mathcal{C}}$ are equal for all $t \in \mathbb{R}_{\geq 0}$ and the same holds for their limits if $t \rightarrow \infty$. This completes the proof as for $u \sim v$ we obtain $u \models \mathrm{L} \sqsubseteq p \Psi$ iff $[u] \models \mathrm{L} \sqsubseteq p \Psi$ iff $[v] \models \mathrm{L} \sqsubseteq p \Psi$ iff $v \models \mathrm{L}^{\sqsubseteq p} \Psi$.

This theorem shows that bisimilar states satisfy the same CSL formulas. The reverse direction, however, does not hold in general. One reason is obvious: In this paper we use a purely state-based logic whereas our definition of strong bisimulation also accounts for action names. Therefore it comes to no surprise that CSL cannot characterize strong bisimulation. However, there is another more profound reason which is analogous to the discrete-time setting where extensions of PCTL to Markov decision processes [28,4] also cannot express strong bisimilarity: CSL and PCTL only allow to specify infima and suprema as probability bounds under a denumerable class of randomized schedulers; therefore intuitively, CSL cannot characterize exponential distributions which neither contribute to the supremum nor to the infimum of the probability measures of a given set of paths. Thus the counterexample from [4, Fig 9.5] interpreted as a CTMDP applies verbatim to our case.

\section{Conclusion}

In this paper we define strong bisimulation on CTMDPs and propose a nondeterministic extension of CSL to CTMDP that allows to express a wide class of performance and dependability measures. Using a measure-theoretic argument we prove our logic to be well-defined. Our main contribution is the proof that strong bisimilarity preserves the validity of CSL formulas. However, our logic is not capable of characterizing strong bisimilarity. To this end, action-based logics provide a natural starting point.

Acknowledgements This research has been performed as part of the QUPES project that is financed by the Netherlands Organization for Scientific Research (NWO). Daniel Klink and David N. Jansen are kindly acknowledged for many fruitful discussions. 


\section{References}

1. Abdeddaïm, Y., Asarin, E., Maler, O.: On optimal scheduling under uncertainty. In: TACAS. LNCS, Vol. 2619. Springer (2003) 240-253

2. Ash, R. B., Doléans-Dade, C. A.: Probability $\&$ Measure Theory. 2nd edn. Academic Press (2000)

3. Aziz, A., Sanwal, K., Singhal, V., Brayton, R. K.: Model-checking continous-time Markov chains. ACM Trans. Comput. Log. 1 (2000) 162-170

4. Baier, C.: On Algorithmic Verification Methods for Probabilistic Systems. Habilitation Thesis (1998) University of Mannheim.

5. Baier, C., Haverkort, B. R., Hermanns, H., Katoen, J.-P.: Model-checking algorithms for continuous-time Markov chains. IEEE TSE 29 (2003) 524-541

6. Baier, C., Haverkort, B. R., Hermanns, H., Katoen, J.-P.: Nonuniform CTMDPs. unpublished manuscript (2004)

7. Baier, C., Hermanns, H., Katoen, J.-P., Haverkort, B. R.: Efficient computation of timebounded reachability probabilities in uniform continuous-time Markov decision processes. Theor. Comp. Sci. 345 (2005) 2-26

8. Baier, C., Katoen, J.-P., Hermanns, H., Wolf, V.: Comparative branching-time semantics for Markov chains. Information and Computation 200 (2005) 149-214

9. Baier, C., Kwiatkowska, M. Z.: Model checking for a probabilistic branching time logic with fairness. Distr. Comp. 11 (1998) 125-155

10. Beutler, F. J., Ross, K. W.: Optimal policies for controlled Markov chains with a constraint. Journal of Mathematical Analysis and Appl. 112 (1985) 236-252

11. Bianco, A., de Alfaro, L.: Model checking of probabilistic and nondeterministic systems. In: FSTTCS. LNCS, Vol. 1026. Springer (1995) 499-513

12. Boudali, H., Crouzen, P., Stoelinga, M. I. A.: Dynamic fault tree analysis using input/output interactive Markov chains. In: Dependable Systems and Networks. IEEE (2007) 708-717

13. Buchholz, P.: Exact and ordinary lumpability in finite Markov chains. Journal of Applied Probability 31 (1994) 59-75

14. Chiola, G., Marsan, M. A., Balbo, G., Conte, G.: Generalized stochastic Petri nets: A definition at the net level and its implications. IEEE TSE 19 (1993) 89-107

15. de Alfaro, L.: Formal Verification of Probabilistic Systems. PhD thesis, Stanford University (1997)

16. Desharnais, J., Panangaden, P.: Continuous stochastic logic characterizes bisimulation of continuous-time Markov processes. Journal of Logic and Algebraic Programming 56 (2003) 99-115

17. Feinberg, E. A.: Continuous time discounted jump Markov decision processes: A discreteevent approach. Mathematics of Operations Research 29 (2004) 492-524

18. Givan, R., Dean, T., Greig, M.: Equivalence notions and model minimization in Markov decision processes. Artificial Intelligence 147 (2003) 163-223

19. Hermanns, H.: Interactive Markov Chains: The Quest for Quantified Quality. LNCS, Vol. 2428. Springer (2002)

20. Howard, R. A.: Dynamic Probabilistic Systems. John Wiley and Sons (1971)

21. Katoen, J.-P., Klink, D., Leucker, M., Wolf, V.: Three-valued abstraction for continuoustime Markov chains. In: CAV. LNCS, Vol. 4590. Springer (2007) 316-329

22. Kemeny, J. G., Snell, J. L., Knapp, A. W.: Denumerable Markov Chains. 2nd edn. Springer (1976)

23. Larsen, K. G., Skou, A.: Bisimulation through probabilistic testing. Information and Computation 94 (1991) 1-28

24. López, G. G. I., Hermanns, H., Katoen, J.-P.: Beyond memoryless distributions: Model checking semi-Markov chains. In: PAPM-PROBMIV. LNCS, Vol. 2165. Springer (2001) $57-70$

25. Puterman, M. L.: Markov Decision Processes: Discrete Stochastic Dynamic Programming. John Wiley and Sons (1994)

26. Qiu, Q., Pedram, M.: Dynamic power management based on continuous-time Markov decision processes. In: DAC. ACM Press (1999) 555-561

27. Sanders, W. H., Meyer, J. F.: Stochastic activity networks: Formal definitions and concepts. In: FMPA. LNCS, Vol. 2090. Springer (2000) 315-343 
28. Segala, R., Lynch, N.: Probabilistic simulations for probabilistic processes. Nordic Journal of Computing 2 (1995) 250-273

29. Vardi, M. Y.: Automatic verification of probabilistic concurrent finite-state programs. In: FOCS. IEEE (1985) 327-338

30. Wolovick, N., Johr, S.: A characterization of meaningful schedulers for continuous-time Markov decision processes. In: FORMATS. LNCS, Vol. 4202. Springer (2006) 352-367 


\section{Aachener Informatik-Berichte}

This list contains all technical reports published during the past five years. A complete list of reports dating back to 1987 is available from http://aib.informatik.rwthaachen.de/. To obtain copies consult the above URL or send your request to: Informatik-Bibliothek, RWTH Aachen, Ahornstr. 55, 52056 Aachen, Email: biblio@informatik.rwth-aachen.de

2001-01 * Jahresbericht 2000

2001-02 Benedikt Bollig, Martin Leucker: Deciding LTL over Mazurkiewicz Traces

2001-03 Thierry Cachat: The power of one-letter rational languages

2001-04 Benedikt Bollig, Martin Leucker, Michael Weber: Local Parallel Model Checking for the Alternation Free mu-Calculus

2001-05 Benedikt Bollig, Martin Leucker, Thomas Noll: Regular MSC Languages

2001-06 Achim Blumensath: Prefix-Recognisable Graphs and Monadic SecondOrder Logic

2001-07 Martin Grohe, Stefan Wöhrle: An Existential Locality Theorem

2001-08 Mareike Schoop, James Taylor (eds.): Proceedings of the Sixth International Workshop on the Language-Action Perspective on Communication Modelling

2001-09 Thomas Arts, Jürgen Giesl: A collection of examples for termination of term rewriting using dependency pairs

2001-10 Achim Blumensath: Axiomatising Tree-interpretable Structures

2001-11 Klaus Indermark, Thomas Noll (eds.): Kolloquium Programmiersprachen und Grundlagen der Programmierung

2002-01* Jahresbericht 2001

2002-02 Jürgen Giesl, Aart Middeldorp: Transformation Techniques for ContextSensitive Rewrite Systems

2002-03 Benedikt Bollig, Martin Leucker, Thomas Noll: Generalised Regular MSC Languages

2002-04 Jürgen Giesl, Aart Middeldorp: Innermost Termination of ContextSensitive Rewriting

2002-05 Horst Lichter, Thomas von der Maßen, Thomas Weiler: Modelling Requirements and Architectures for Software Product Lines

2002-06 Henry N. Adorna: 3-Party Message Complexity is Better than 2-Party Ones for Proving Lower Bounds on the Size of Minimal Nondeterministic Finite Automata

2002-07 Jörg Dahmen: Invariant Image Object Recognition using Gaussian Mixture Densities

2002-08 Markus Mohnen: An Open Framework for Data-Flow Analysis in Java

2002-09 Markus Mohnen: Interfaces with Default Implementations in Java

2002-10 Martin Leucker: Logics for Mazurkiewicz traces

2002-11 Jürgen Giesl, Hans Zantema: Liveness in Rewriting

2003-01* Jahresbericht 2002

2003-02 Jürgen Giesl, René Thiemann: Size-Change Termination for Term Rewriting

2003-03 Jürgen Giesl, Deepak Kapur: Deciding Inductive Validity of Equations

2003-04 Jürgen Giesl, René Thiemann, Peter Schneider-Kamp, Stephan Falke: Improving Dependency Pairs

2003-05 Christof Löding, Philipp Rohde: Solving the Sabotage Game is PSPACEhard

2003-06 Franz Josef Och: Statistical Machine Translation: From Single-Word Models to Alignment Templates

2003-07 Horst Lichter, Thomas von der Maßen, Alexander Nyßen, Thomas Weiler: Vergleich von Ansätzen zur Feature Modellierung bei der Softwareproduktlinienentwicklung

2003-08 Jürgen Giesl, René Thiemann, Peter Schneider-Kamp, Stephan Falke: Mechanizing Dependency Pairs 
2004-01 * Fachgruppe Informatik: Jahresbericht 2003

2004-02 Benedikt Bollig, Martin Leucker: Message-Passing Automata are expressively equivalent to EMSO logic

2004-03 Delia Kesner, Femke van Raamsdonk, Joe Wells (eds.): HOR 2004 - 2nd International Workshop on Higher-Order Rewriting

2004-04 Slim Abdennadher, Christophe Ringeissen (eds.): RULE 04 - Fifth International Workshop on Rule-Based Programming

2004-05 Herbert Kuchen (ed.): WFLP 04 - 13th International Workshop on Functional and (Constraint) Logic Programming

2004-06 Sergio Antoy, Yoshihito Toyama (eds.): WRS 04 - 4th International Workshop on Reduction Strategies in Rewriting and Programming

2004-07 Michael Codish, Aart Middeldorp (eds.): WST 04 - 7th International Workshop on Termination

2004-08 Klaus Indermark, Thomas Noll: Algebraic Correctness Proofs for Compiling Recursive Function Definitions with Strictness Information

2004-09 Joachim Kneis, Daniel Mölle, Stefan Richter, Peter Rossmanith: Parameterized Power Domination Complexity

2004-10 Zinaida Benenson, Felix C. Gärtner, Dogan Kesdogan: Secure MultiParty Computation with Security Modules

2005-01* Fachgruppe Informatik: Jahresbericht 2004

2005-02 Maximillian Dornseif, Felix C. Gärtner, Thorsten Holz, Martin Mink: An Offensive Approach to Teaching Information Security: "Aachen Summer School Applied IT Security"

2005-03 Jürgen Giesl, René Thiemann, Peter Schneider-Kamp: Proving and Disproving Termination of Higher-Order Functions

2005-04 Daniel Mölle, Stefan Richter, Peter Rossmanith: A Faster Algorithm for the Steiner Tree Problem

2005-05 Fabien Pouget, Thorsten Holz: A Pointillist Approach for Comparing Honeypots

2005-06 Simon Fischer, Berthold Vöcking: Adaptive Routing with Stale Information

2005-07 Felix C. Freiling, Thorsten Holz, Georg Wicherski: Botnet Tracking: Exploring a Root-Cause Methodology to Prevent Distributed Denial-ofService Attacks

2005-08 Joachim Kneis, Peter Rossmanith: A New Satisfiability Algorithm With Applications To Max-Cut

2005-09 Klaus Kursawe, Felix C. Freiling: Byzantine Fault Tolerance on General Hybrid Adversary Structures

2005-10 Benedikt Bollig: Automata and Logics for Message Sequence Charts

2005-11 Simon Fischer, Berthold Vöcking: A Counterexample to the Fully Mixed Nash Equilibrium Conjecture

2005-12 Neeraj Mittal, Felix Freiling, S. Venkatesan, Lucia Draque Penso: Efficient Reductions for Wait-Free Termination Detection in Faulty Distributed Systems

2005-13 Carole Delporte-Gallet, Hugues Fauconnier, Felix C. Freiling: Revisiting Failure Detection and Consensus in Omission Failure Environments

2005-14 Felix C. Freiling, Sukumar Ghosh: Code Stabilization

2005-15 Uwe Naumann: The Complexity of Derivative Computation

2005-16 Uwe Naumann: Syntax-Directed Derivative Code (Part I: TangentLinear Code)

2005-17 Uwe Naumann: Syntax-directed Derivative Code (Part II: Intraprocedural Adjoint Code)

2005-18 Thomas von der Maßen, Klaus Müller, John MacGregor, Eva Geisberger, Jörg Dörr, Frank Houdek, Harbhajan Singh, Holger Wußmann, Hans-Veit Bacher, Barbara Paech: Einsatz von Features im SoftwareEntwicklungsprozess - Abschlußbericht des GI-Arbeitskreises "Features"

2005-19 Uwe Naumann, Andre Vehreschild: Tangent-Linear Code by Augmented LL-Parsers 
2005-20 Felix C. Freiling, Martin Mink: Bericht über den Workshop zur Ausbildung im Bereich IT-Sicherheit Hochschulausbildung, berufliche Weiterbildung, Zertifizierung von Ausbildungsangeboten am 11. und 12. August 2005 in Köln organisiert von RWTH Aachen in Kooperation mit BITKOM, BSI, DLR und Gesellschaft fuer Informatik (GI) e.V.

2005-21 Thomas Noll, Stefan Rieger: Optimization of Straight-Line Code Revisited

2005-22 Felix Freiling, Maurice Herlihy, Lucia Draque Penso: Optimal Randomized Fair Exchange with Secret Shared Coins

2005-23 Heiner Ackermann, Alantha Newman, Heiko Röglin, Berthold Vöcking: Decision Making Based on Approximate and Smoothed Pareto Curves

2005-24 Alexander Becher, Zinaida Benenson, Maximillian Dornseif: Tampering with Motes: Real-World Physical Attacks on Wireless Sensor Networks

2006-01 * Fachgruppe Informatik: Jahresbericht 2005

2006-02 Michael Weber: Parallel Algorithms for Verification of Large Systems

2006-03 Michael Maier, Uwe Naumann: Intraprocedural Adjoint Code Generated by the Differentiation-Enabled NAGWare Fortran Compiler

2006-04 Ebadollah Varnik, Uwe Naumann, Andrew Lyons: Toward Low Static Memory Jacobian Accumulation

2006-05 Uwe Naumann, Jean Utke, Patrick Heimbach, Chris Hill, Derya Ozyurt, Carl Wunsch, Mike Fagan, Nathan Tallent, Michelle Strout: Adjoint Code by Source Transformation with OpenAD/F

2006-06 Joachim Kneis, Daniel Mölle, Stefan Richter, Peter Rossmanith: Divideand-Color

2006-07 Thomas Colcombet, Christof Löding: Transforming structures by set interpretations

2006-08 Uwe Naumann, Yuxiao Hu: Optimal Vertex Elimination in SingleExpression-Use Graphs

2006-09 Tingting Han, Joost-Pieter Katoen: Counterexamples in Probabilistic Model Checking

2006-10 Mesut Günes, Alexander Zimmermann, Martin Wenig, Jan Ritzerfeld, Ulrich Meis: From Simulations to Testbeds - Architecture of the Hybrid MCG-Mesh Testbed

2006-11 Bastian Schlich, Michael Rohrbach, Michael Weber, Stefan Kowalewski: Model Checking Software for Microcontrollers

2006-12 Benedikt Bollig, Joost-Pieter Katoen, Carsten Kern, Martin Leucker: Replaying Play in and Play out: Synthesis of Design Models from Scenarios by Learning

2006-13 Wong Karianto, Christof Löding: Unranked Tree Automata with Sibling Equalities and Disequalities

2006-14 Danilo Beuche, Andreas Birk, Heinrich Dreier, Andreas Fleischmann, Heidi Galle, Gerald Heller, Dirk Janzen, Isabel John, Ramin Tavakoli Kolagari, Thomas von der Maßen, Andreas Wolfram: Report of the GI Work Group "Requirements Management Tools for Product Line Engineering"

2006-15 Sebastian Ullrich, Jakob T. Valvoda, Torsten Kuhlen: Utilizing optical sensors from mice for new input devices

2006-16 Rafael Ballagas, Jan Borchers: Selexels: a Conceptual Framework for Pointing Devices with Low Expressiveness

2006-17 Eric Lee, Henning Kiel, Jan Borchers: Scrolling Through Time: Improving Interfaces for Searching and Navigating Continuous Audio Timelines

2007-01 * Fachgruppe Informatik: Jahresbericht 2006

2007-02 Carsten Fuhs, Jürgen Giesl, Aart Middeldorp, Peter Schneider-Kamp, René Thiemann, and Harald Zankl: SAT Solving for Termination Analysis with Polynomial Interpretations

2007-03 Jürgen Giesl, René Thiemann, Stephan Swiderski, and Peter SchneiderKamp: Proving Termination by Bounded Increase

2007-04 Jan Buchholz, Eric Lee, Jonathan Klein, and Jan Borchers: coJIVE: A System to Support Collaborative Jazz Improvisation 
2007-05 Uwe Naumann: On Optimal DAG Reversal

2007-06 Joost-Pieter Katoen, Thomas Noll, and Stefan Rieger: Verifying Concurrent List-Manipulating Programs by LTL Model Checking

2007-07 Alexander Nyßen, Horst Lichter: MeDUSA - MethoD for UML2-based Design of Embedded Software Applications

2007-08 Falk Salewski and Stefan Kowalewski: Achieving Highly Reliable Embedded Software: An empirical evaluation of different approaches

2007-09 Tina Kraußer, Heiko Mantel, and Henning Sudbrock: A Probabilistic Justification of the Combining Calculus under the Uniform Scheduler Assumption

2007-12 Uwe Naumann: An L-Attributed Grammar for Adjoint Code

2007-13 Uwe Naumann, Michael Maier, Jan Riehme, and Bruce Christianson: Second-Order Adjoints by Source Code Manipulation of Numerical Programs

2007-14 Jean Utke, Uwe Naumann, Mike Fagan, Nathan Tallent, Michelle Strout, Patrick Heimbach, Chris Hill, and Carl Wunsch: OpenAD/F: A Modular, Open-Source Tool for Automatic Differentiation of Fortran Codes

* These reports are only available as a printed version.

Please contact biblio@informatik.rwth-aachen. de to obtain copies. 